
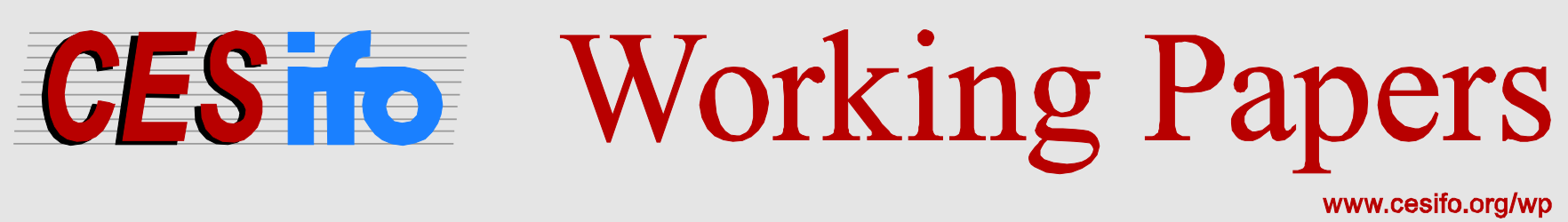

\title{
Cake-Eating with Private Information
}

\author{
Reyer Gerlagh \\ Matti Liski
}

\author{
CESIFO WORKING PAPER NO. 5050 \\ CATEGORY 9: RESOURCE AND ENVIRONMENT ECONOMICS \\ NOVEMBER 2014
}

An electronic version of the paper may be downloaded

- from the SSRN website:

- from the RePEc website:

- from the CESifo website:

WWW.SSRN.com

www.RePEc.org

www.CESifo-group.org/wp

\section{CESifo}




\title{
Cake-Eating with Private Information
}

\begin{abstract}
We consider a model of cake-eating with private information. The model captures phenomena such as trust and "security of supply" in resource-use relationships. It also predicts supply shocks as an equilibrium phenomenon: privately informed sellers have incentives to reveal resource scarcity too late, through a supply disruption, after which they exploit the consumers' inability to immediately adjust demand. Two puzzles that a standard exhaustibleresource theory cannot explain are resolved: sellers have an incentive to overstate their resources rather than emphasize scarcity, and consumers can switch to alternatives before exhausting the resource thereby leaving socially valuable resource in the ground.
\end{abstract}

JEL-Code: D400, D900, O330, Q400.

Keywords: exhaustible resources, asymmetric information, resource dependence.

Reyer Gerlagh

Tilburg University

Economics Department

The Netherlands

r.gerlagh@uvt.nl
Matti Liski

Aalto University

Economics Department

Finland

matti.liski@aalto.fi

Oct 20, 2014

We have presented working paper versions at seminars in Helsinki, LSE, Madrid, Munich, Tilburg, Toulouse and in ESEM, EAERE and SURED conferences; we thank the participants for many comments and discussions. In particular, we thank Philippe Mahenc and Juuso Välimäki. 


\section{Introduction}

We consider an exhaustible-resource market where the owner of the resource is privately informed about the reserves, that is, how much cumulative consumption the resource can offer. The buyer wishes to consume the resource and, ultimately, when the resource is depleted, move to an alternative source of consumption. The alternative offers an opportunity surplus to the consumer, but it becomes available with a delay and therefore its adoption should be decided before the resource runs out. The seller has no direct means for a verifiable communication of its holdings but can indirectly convey information through its actions in the market. Without precise knowledge of the seller's endowment, the consumer's decision for continuation of resource dependence, or alternatively stopping the relationship, thus depends on the observed terms of trade in the market.

In the equilibrium that we consider, a sufficiently large seller communicates a "secure supply" through prices sufficiently low, in the sense that a critically small resource owner could not replicate the offer. However, since the resource is finite, ultimately all seller types become small. The privately informed seller gains from revealing too late - from the consumer's perspective - that it is running out of stock. The seller will, ultimately, exploit the buyer's inability to move to the substitute immediately: the seller reveals scarcity - it becomes public information - through a disruption in supply. A supply shock is thus an equilibrium phenomenon. The buyer side rationally accepts the chance of being exploited but requires a compensation for accepting this risk, through generous terms of trade (low resource prices), prior to the shock. The equilibrium describes a tradeoff between the seller's incentive to benefit from the final scarcity and the consumer's demand for a supply that matches the substitute surplus, and additionally compensates for the risk of a supply disruption. Resource supplies are higher for more pessimistic reserve estimates on the consumer side - larger supplies justify continuing the relationship and accepting a larger potential supply disruption. Yet, the equilibrium also describes the possibilty of too early transitions to the resource substitute. A sufficiently good outside option or a favorable change in the outside option can lead the buyer to move away from the resource, even though, in expectations, some socially valuable resource will be left in the ground.

The exhaustible-resource problem with private information opens fundamental questions for the resource-use theory - yet, the problem has not been explored before. The model developed here can shed light on two phenomena in resource-use relationships that the standard resource theory cannot explain. First, our model describes "demand 
management" motives on the seller side where large reserves are emphasized rather than scarcity. Second, our model rationalizes "caution" on the consumer side that can lead to transitions away from resources before exhaustion.

An illuminating example for the second phenomenon can be found in the World's transition away from the natural nitrogen supplies. At the turn of the 20th century, agricultural nitrogen became a key scarce natural resource commodity in Europe, leading Sir William Crookes, the president of the British Association for the Advancement of Science, in 1889 to appeal to chemists to develop a synthetic solution to the nitrogen problem, as otherwise "All England and all civilized nations stand in deadly peril of not having enough to eat", potentially as early as in the 1930's. The early industrialized nations had become critically dependent on the deposits of natural sodium nitrogen from the Atacama desert of Chile. ${ }^{1}$ Chile was the sole supplier of this commodity in four decades until the 1920's. Then, a synthetic substitute was derived through the HaberBosch process, named after the two Nobel Prize winners who developed the process that turned out to be "[...] one of the most important inventions in the chemical industry ever." (Mokyr, 1998). ${ }^{2,3}$ After the innovation, it took more than a decade for the world consumption to depart from the natural supplies. Surprisingly, the monopoly did not only face a competitor but lost its business entirely: a significant fraction of the resource was left unused (Smil, 2001). The resource was relatively easy to extract (Whitbeck, 1931), and, in view of the standard exhaustible-resource theory (Dasgupta and Heal, 1979), it is unexpected that a costly substitute made a relatively homogenous resource obsolete. Given that the valuable resource was left unused, it seems that the adoption of the substitute technology was too much hurried.

Moving hundred years in time, to the present-day resource relationships, the phenomenon of demand management appears as a puzzle not described by existing resourceuse theory. The following headline from the Telegraph of March 22, 2013 is revealing: ${ }^{4}$

"The world's oil reserves have been exaggerated by up to a third."

Or from The Huffington Post September 2, 2011:

\footnotetext{
${ }^{1}$ For the fascinating history of nitrogen use, natural fixation and synthetic production, see, e.g., Leigh (2004) and Smil (2001).

${ }^{2}$ Whitbeck (1931) provides a succinct description of the resource reserve, its exploitation technology, costs, production numbers, as well as the basic facts of the substitute entry.

${ }^{3}$ See Montéon (1975) for the role of British capital in the resource exploitation, and, e.g., Brown (1963) for the Chilean government's resource-use policies.

${ }^{4}$ These headlines are obtained through a simple Google search. A more systematic coverage of the concerns regarding the size of the Saudi reserves is in Simmons (2005).
} 
"Wikileaks Cable: Saudi Oil Reserves Exaggerated By 40 Percent."

Why would dominant resource sellers exaggerate their reserves? Prices increase with the perceived scarcity; if anything, resource theory following Hotelling (1931), suggests that dominant resource sellers should underestimate rather than overestimate their resource holdings. The current theory has no hope of explaining why the dominant resource sellers often communicate with the market to emphasize stability and the security of supply. ${ }^{5}$

The nitrogen and oil examples illustrate a common theme. When planning for the use of resources and future dependence on them, it is essential to take account of the fact that we do not know how long resources will last precisely. Yet few studies of exhaustible-resource allocations over time give this problem due consideration. Pindyck (1980) and others have analyzed the uncertainty of future resource reserves but, for one reason or other, they did not expound on resource uncertainties arising from asymmetric information, and on how such asymmetries in uncertainties can lead to drastic changes in behavior. ${ }^{6}$ The resource theorists seem to have disregarded the problem altogether. The standard resource-use models fail to capture the essence of the buyer-seller relationships illustrated above - hurried transitions away from resources, as well as "demand management" motives on the seller side. We intend to go to the other extreme; we concentrate entirely on uncertainties arising from privately informed resource owners and ignore the uncertainties that a resource market must normally cope with.

In this paper, we develop a simple model of resource dependence to capture exactly these features. We have two strategic parties but the bargaining is not explicit as the resource is traded in the market rather than in a direct bilateral relationship. The timing assumptions that we make support a market interpretation of the resource relationship and an implicit nature of bargaining. The relationship description follows Gerlagh and Liski (2011), but the informational asymmetries introduced in the current paper are novel and necessary for the two features of resource markets outlined above. ${ }^{7}$ While not cheap

\footnotetext{
${ }^{5}$ To illustrate: "We've got almost 30 percent of the world's oil. For us, the objective is to assure that oil remains an economically competitive source of energy. Oil prices that are too high reduce demand growth for oil and encourage the development of alternative energy sources" (Adel al-Jubeir, foreign policy adviser of crown prince Abdullah of Saudi Arabia, Herald Tribune, Jan 24, 2007).

${ }^{6}$ While Pindyck (1980) considers multiple uncertainties, there is a literature that seeks to answer the question "How to eat a cake of an unknown size"; see Kemp (1976), and, for example, Kumar (2005). There are no private information considerations in this literature.

${ }^{7}$ To be explicit, the two puzzles presented - the consumer side caution and the seller side overreporting of reserves - cannot be addressed without hidden information. In fact, we were initially
} 
talk (Crawford and Sobel, 1982), the periodic interactions we describe between resource sellers and buyers has an element thereof: the seller offers supplies to influence beliefs and, then, if the buyer continues without investing, the offer is implicitly accepted and supply is delivered; if the offer is "declined" and investment into a substitute takes place, then the seller does not have to deliver the supply offer. These staged interactions preserve a non-trivial sharing of the surplus, depending on the primitives such as adjustment delays of the demand, resource size, and the surplus from options outside the relationship.

The setting shares similarities with the literature on the Coase conjecture - Hörner and Kamien (2004) establish that the resource monopsony problem is equivalent to the durable-good monopoly problem. ${ }^{8}$ However, our setting is a bilateral monopoly with dynamic signaling (Fudenberg and Tirole, 1983) with a different strategic variable (stopping decision with delay), leading to quite different equilibrium outcomes. In particular, the informed agent takes initiative in the relationship. After all, in reality, it is the resource seller who strategically interacts with the market. The individual resource consumers play no strategic role, but the buyer's agent (government) can respond to the information generated through the market interaction through investment in a substitute if such is deemed appropriate. ${ }^{9}$

We describe a stationary equilibrium in a situation where the informed agent (seller) takes initiative by offering terms of trade to the market, and the uninformed agent (buyer) decides whether to continue the relationship. While this timing takes us to the domain of dynamic signaling, and thus leads to multiplicity of sequential equilibria (Fudenberg

interested in the asymmetric information problem in resource extraction; the full information results in Gerlagh and Liski (2011) were necessary steps for developing a tractable model for the setting with hidden information.

${ }^{8}$ There is a long tradition in resource economics to study the strategic interactions in the resource markets, although the formal connection to the durable-good theory was first presented by Hörner and Kamien (2004). There are two branches of literature that are Coasian in spirit: the optimal tariff literature (e.g., Newbery, 1983; Maskin and Newbery,1990; see Karp and Newbery 1993 for a review); and the literature on strategic R\&D and technology adoption in exhaustible-resource markets (Dasgupta et al., 1983; Gallini et al., 1983, and Hoel, 1983; Lewis et al.,1986; Harris and Vickers 1995, Harstad and Liski (2013)). The common theme in this literature is that the co-ordinated action on the buyer side can be used to decrease the seller's resource rent. None of these papers consider asymmetric information.

${ }^{9}$ In a typical durable-good problem, the uninformed agent makes repeated offers to the informed agent whose valuation is private information (see, e.g., Gul et al. 1986). Assuming screening of the seller by the uninformed buyer would be at odds with the market interaction. This interpretation would be difficult to achieve under a structure where the uninformed agent takes initiative in screening the informed agent. Deneckere and Liang (2006) consider screening, which is more natural in their case since there is no market involved. 
and Tirole 1983; see also Ausubel et al., 2002), the structure of stationary equilibria is relatively simple due to the nature of the buyer's stopping problem. In fact, we make assumptions that ensure existence of stationarity in terms of resource stock beliefs: under continuation, the buyer updates beliefs of the seller's size upwards at the same rate as the resource stock is exhausted. This allows a relatively simple analysis while keeping the substance-related key concepts in the analysis, such as the resource scarcity, substitute surplus, and the determinants of the resource dependence. Clearly, we cannot make claims regarding generality, but we have elaborated a number of extensions that will be discussed.

There is a large literature on dynamic models of adverse selection and signaling, building on the static models of Akerlof (1970) and Spence (1973), respectively. The dynamic extensions of the static model by Spence introduce time for changing the information exchange and commitment assumptions. ${ }^{10}$ In contrast, our paper contributes to the signaling literature by developing a tractable approach with private information where dynamics arise from changes in a physical state. Our approach to dynamics is very different and motivated by the physical nature of the problem. In our dynamic equilibrium, the seller's type is revealed either by the buyer's irreversible action to stop the relationship, or if consumption continues, the seller will reveal its type by separating at a future time that is known to the seller but random from the buyer's point of view. Thus, by stopping, the buyer can verify the seller's type but, since the action is irreversible, the buyer would like to know the type before making the decision, as stopping may leave some socially valuable stock in the ground. Under continuation, our equilibrium is neither a traditional pooling nor separation equilibrium: sellers pool as long as the game continues but each type has a privately known and stock-dependent opt-out time from the equilibrium. These features are novel and arise from real changes in the type space and not from dynamic information exchange per se.

The paper is organized as follows. In the next Section, we introduce the formal notation and present the basic assumptions regarding the strategic interaction as well as restrictions on beliefs. We state the conjectured equilibrium, and the main Theorem for the existence and structure of the equilibrium. In Section 3, we present the buyer's and

\footnotetext{
${ }^{10}$ In Nöldeke and Van Damme (1990), the privately informed seller has a sequence of opportunities for trading and signaling; the separation of seller types can be obtained through off-equilibrium beliefs when the opportunities for information exchange increase without bound. Swinkels (1999) shows that the results depend critically on whether the offers are private or public; Kremer and Skrzypacz (2005) and Daley and Green (2012) both analyze the the effect of exogenous news arrival on equilibrium dynamics.
} 
the seller's dynamic problems, and characterize the equilibrium. Section 4 elaborates on how concepts such as trust, mutual dependence, and supply shocks are captured by the model, through the analysis of the key parameters In Section 5, we introduce changes in the buyer's outside option on the equilibrium path, to identify a source of supply disruption and resource abandoning that is different from that in the main model. Much of the analysis progresses without discussions on the alternative assumptions and connections to the literature; we provide an extensive discussion in Section 6. In particular, the Section demonstrates that the distributional assumptions that facilitate the analysis are not critical for the substantial implications. Section 7 provides concluding remarks on two empirical cases where the results may turn relevant. All proofs are in the Appendix.

\section{The model}

\subsection{Basic setting}

There are two strategic agents: a seller who owns a resource stock and a buyer who wants to consume the resource. Time runs continuously, $t \in[0, \infty)$, and at each $t$ where there is consumption, $q_{t} \geqslant 0$, the buyer enjoys consumption utility, $U\left(q_{t}\right)$, assumed to be an increasing, twice differentiable, and strictly concave function. The seller has full powers to set the unit price of consumption, $p_{t} \geqslant 0$. After the buyer observes $p_{t}$, it can choose to end the relationship, or not. Over time, the economy can be in one of two states: the consumer has either decided to end the relationship in the past, or not. If no stopping decision has been made, the dependence on the seller is strict in the sense that there is no alternative source of consumption currently available; in this state, the quantity consumed at price $p_{t}$ follows from $U^{\prime}\left(q_{t}\right)=p_{t}$.

The consumer can decide to end the resource-consumption relationship at any time, following a protocol defined below; but, after making the decision, the resource is still needed for a known and given time interval of length $k$. Here, $k$ is the time-to-build constraint for the substitute, capturing the degree of resource dependence. Once in place, the substitute replaces the resource irreversibly and generates a constant utility flow that we denote by $\bar{u}$. In the analysis, we consider variations in $k$ and $\bar{u}$, and assume $k \in(0, \infty)$ and $\bar{u} \in(0, \infty)$.

Whether the buyer stops or not at time $t$ is described by the choice $d_{t} \in\{0,1\}$, where $d_{t}=1$ means stopping. The seller setting $p_{t}$ and the buyer choosing $d_{t}$ are the only strategic choices in this game. 
The buyer's problem is that only the seller knows the exact size of the initial stock, $s_{0}>0$. Thus, only the seller knows how much is left after some publicly known cumulative use, $s_{t}=s_{0}-Q_{t} ; \int_{0}^{t} q_{\tau} d \tau=Q_{t}$. We introduce shortly a specific structure for the buyer's belief about the remaining resource stock. When should the consumer initiate the transition to the substitute? For conciseness, we say that the answer to this question defines the stopping time for the resource dependence, although one should bear in mind that the resource is still needed during the transition period of length $k$.

Let us now define the protocol for strategic interactions. Time is continuous but strategic interactions take place at discrete time points in the time line, $t_{i}=\varepsilon i$ where $\varepsilon>0$ and $i=1,2,3, \ldots$ At given $t_{i}$, the buyer has beliefs about the seller's remaining resource stock, formulated shortly. The choices at each $t_{i}$ freeze actions for the next $\varepsilon$ interval of time; below, we let $\varepsilon$ converge to zero to analyze the continuous-time limit. After each $\varepsilon$ units of time, conditional on continuation, the interaction starts anew. The timing of moves at any $t=t_{i}$ where the buyer has not yet "invested" in the past $\left(d_{\tau}=0, \forall \tau<t_{i}\right)$ is:

1. The seller offers supply price $p_{t} \geqslant 0$;

2. The buyer updates beliefs and decides on investment $d_{t} \in\{0,1\}$;

3. If $d_{t}=0$, the seller delivers the demanded $q_{t}$ at price $p_{t}$, and the game continues to stage 1 at $t+\varepsilon$. If $d_{t}=1$, the strategic interaction stops, and the seller offers its privately optimal monopoly price at each $t \in\left[t_{i}, t_{i}+k\right]$.

These timing assumptions create a bargaining situation that sustains a division of surplus dependent on the fundamentals of the problem, even when time discounting is absent, which we assume. Since the buyer can respond to $p_{t}$ in the same period, the seller will have to choose a price that gives the buyer at least the expected surplus achievable from stopping immediately. Figure 1 illustrates the overall timeline.

The seller's profit flow is $\hat{\pi}\left(p_{t}\right)=p_{t} q_{t}\left(p_{t}\right)$ where $q\left(p_{t}\right)$ is the demand function that satisfies $p_{t}=U^{\prime}\left(q\left(p_{t}\right)\right)$ for $q_{t}>0$. For the analysis, it is convenient to work with quantities, and we write $\pi\left(q_{t}\right)=p\left(q_{t}\right) q_{t}$ with the inverse demand $p\left(q_{t}\right)=U^{\prime}\left(q_{t}\right)$ for the resource supply flow $q_{t}>0$. The seller's total payoff at time $t$, when the buyer's stopping decision is made at time $T$, is generated by $\left(p_{\tau}\right)_{T+k \geqslant \tau \geqslant t}$ or, equivalently, by $\left(q_{\tau}\right)_{T+k \geqslant \tau \geqslant t}$ :

$$
V_{t}=\int_{t}^{T+k} \pi\left(q_{\tau}\right) d \tau .
$$




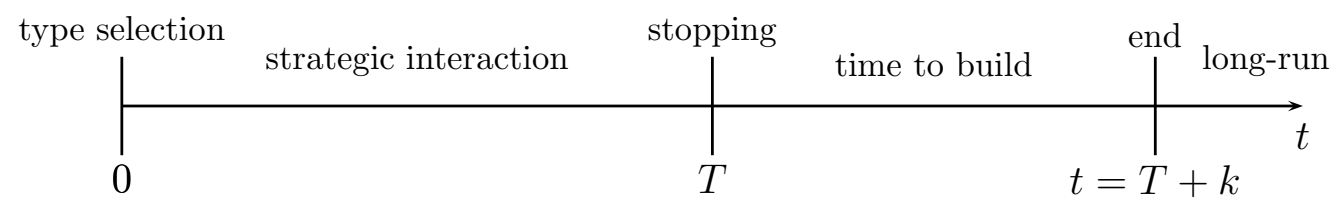

Figure 1: Timeline

The buyer's net surplus flow is the consumer surplus, $u\left(q_{t}\right)=U\left(q_{t}\right)-p\left(q_{t}\right) q_{t}$. We assume that both $u$ and $\pi$ are strictly concave in quantities, and $q^{m}=\operatorname{argmax}\{\pi(q)\}<$ $\infty .{ }^{11}$ Since the consumer is long-lived in this setting, the total consumer surplus, for a path $\left(q_{\tau}\right)_{T+k \geqslant \tau \geqslant t}$, is expressed as an excess surplus over the long-run payoff,

$$
W_{t}=\int_{t}^{T+k}\left[u\left(q_{\tau}\right)-\bar{u}\right] d \tau .
$$

This payoff criterion measures how much more surplus the resource can offer in comparison to the substitute; it coincides with Dutta's (1991) strong long-run average criterion. ${ }^{12}$

\subsection{Stationary strategies}

We are interested in stationary strategies where both the seller's and buyer's strategies are of the cutoff type. The buyer stops the relationship if the offered price is above a given threshold; or, stated in terms of quantities, the buyer will invest in the substitute if the supply falls below a threshold, denoted by $q^{I}>0$. Otherwise, the buyer will not stop and thus continues. Also, the seller types whose resource is above a cutoff size, denoted by $s^{L}$, find it privately optimal to offer a continuation quantity $q^{I}$; smaller seller types will implement stopping. Intuitively, the small types do not have enough resource to meet the buyer's requirement for continuation; but, all types prefer to continue as long as possible since the seller's first-best would be to spread supplies as thinly as possible over time if there was no requirement to supply at least $q^{I}>0$. More precisely, it is common knowledge that there is a "smallest seller" type $s^{L}$ such that only sellers above this type induce continuation: $s_{t} \geq s^{L}$ if and only if $q_{t} \geq q^{I}$. In equilibrium, the beliefs on the buyer's side, under continuation, can be described through the smallest type $s^{L}$.

\footnotetext{
${ }^{11}$ But see Section 6 for a discussion of an extension to a non-concave $u$.

${ }^{12}$ In Appendix, we derive the total consumer surplus expression as a limit of discounted payoff, after introducing the strategies for evaluating the path of the game.
} 
We construct the equilibrium by the guess-and-verify method, assuming that the supply justifying continuation is constant at level $q_{t}=q^{I}$ and then verifying that the best responses on both sides lead to this outcome with consistent beliefs. For beliefs, we consider an exponential prior distribution for seller types, $s_{0} \sim \exp (\alpha)$, with parameter $\alpha \in(0, \infty)$. Given this distribution, the consumer side can form the belief about the smallest seller type $s^{L}$ from the primitives of the setting; we assume the prior is updated to include information about the lowest possible type at time $t=0$, before the first strategic interaction. Beliefs are then fully determined by two parameters: $s^{L}$ for the smallest possible type complying with the consumer's continuation demand $q^{I}$, and the hazard rate $\alpha$ for the distribution. Beliefs in the continuation equilibrium are represented through a density function $f\left(s ; s^{L}, \alpha\right)$, and the corresponding probability that the resource stock falls short of $s$ is given by the cumulative distribution,

$$
F\left(s ; s^{L}, \alpha\right)=\left\{\begin{array}{c}
1-e^{-\alpha\left(s-s^{L}\right)} \text { if } s>s^{L} \\
0 \text { otherwise. }
\end{array}\right.
$$

Stationarity in this sense means that the equilibrium belief remains constant as long as the relationship continues, and will change only when the seller side stops supplying the required quantity. When consuming at rate $q_{t}$ at time $t$, beliefs about the remaining stock should be revised downwards because the true initial stock drifts down at this rate. Yet, in the continuation of the game, the buyer continuously learns that the seller is not of the smallest type, implying an upward drift in beliefs. In a stationary equilibrium, this upward drift in beliefs exactly equals the rate at which the physical stock declines so that the equilibrium beliefs remain stationary. ${ }^{13}$

Note also that while, under continuation, the equilibrium beliefs and actions remain stationary, the true physical stock is gradually depleted: the relationship is expected to end in finite time in equilibrium.

To support such stationary continuation beliefs, the buyer must thus continuously learn that the seller is not of the smallest type. We look for a stationary strategy for the seller, that is, a function that maps from the remaining stock $s_{t}$ to a supply $q_{t}$, given the publicly known belief: $q_{t}=\eta\left(s_{t}, s^{L}\right) \geq 0$, where we suppressed the exogenous parameter $\alpha$ in our notation of beliefs. The buyer's strategy is then, given belief $s^{L}$, a

\footnotetext{
${ }^{13}$ Notice that the type expectation follows a semi-exponential distribution. While $\alpha$ is a constant, determined by the initial distribution, the lower bound $s^{L}$ is an endogenous characteristic of the equilibrium. Both a lower value for $s^{L}$, and a higher value for $\alpha$ represent more pessimistic beliefs about the resource stock, as $\mathbb{E}[s]=s^{L}+1 / \alpha$. A larger value for $\alpha$ represents both a more pessimistic view, but also a lower degree of asymmetry in information, as $\operatorname{Var}[s]=1 / \alpha^{2}$.
} 
function $d_{t}=\mu\left(q_{t}, s^{L}\right) \in\{0,1\}$, i.e., a function that maps the possible supply levels to a decision to continue or stop. The buyer's strategy is described through a cutoff $q^{I}$ so that $d_{t}=\mu\left(q_{t}, s^{L}\right)=1$ iff $q_{t}<q^{I}\left(s^{L}\right)$ : the buyer will invest if the seller's offer falls short of the required supply $q^{I}$, which is dependent on beliefs.

When the seller supplies more than required (off-the-equilibrium), beliefs remain as in equilibrium. The assumption of passive conjectures supports the equilibrium described by constant beliefs $s^{L}$ and threshold policy $q^{I}$; it rules out threats built into the outof-equilibrium beliefs that could lead to intricate history-dependent dynamics in this resource extraction game. For stationary beliefs, each seller type can simply monitor the dynamic depletion of the privately known stock, and at each point in time can decide whether it is profitable to offer $q_{t}=q^{I}$, or not.

\subsection{Conjectured equilibrium}

The stationary equilibrium has a simple structure. The key parameters of the model are $k \in(0, \infty)$ (degree of dependence), $\bar{u} \in(0, \infty)$ (outside option), and $\alpha \in(0, \infty)$ (expectation of scarcity). We denote this parameter set by $\Omega$.

Theorem 1 For any given $(k, \bar{u}, \alpha) \in \Omega$, the stationary equilibrium outcome is one of two possibilities, continuation until the seller induces stopping at a privately-known time or immediate stopping by the consumer:

- (continuation) there is a unique stationary pair of beliefs and supplies $\left(\hat{s}^{L}, \hat{q}^{I}\right)$ such that the buyer is indifferent between continuation and stopping, and the seller strictly prefers continuation until $s_{t} \leq \hat{s}^{L}$, after which the seller supplies $q_{t}<\hat{q}^{I}$ and the buyer finds it optimal to invest;

- (immediate stopping) there is no belief $s^{L}$ supporting a stationary continuation equilibrium; the buyer finds it optimal to invest at $t=0$.

In Fig. 2, we show "demand" and "supply" schedules, with the intersection identifying the equilibrium belief and supply $\left(\hat{s}^{L}, \hat{q}^{I}\right)$. The demand, denoted by $q^{I}=\mathcal{D}\left(s^{L}\right)$, is the quantity $q^{I}$ demanded by the consumer for continuation when $s^{L}$ is the belief on how small the smallest continuing seller can be; it is downward sloping in the belief since (as we establish later) continuing the relationship is less costly when the resource is expected to be larger. The demand schedule is defined only above a critical belief level $s^{*}$; for worse beliefs, the buyer always stops without considering the seller's offer. With increasing $s^{L}$, 


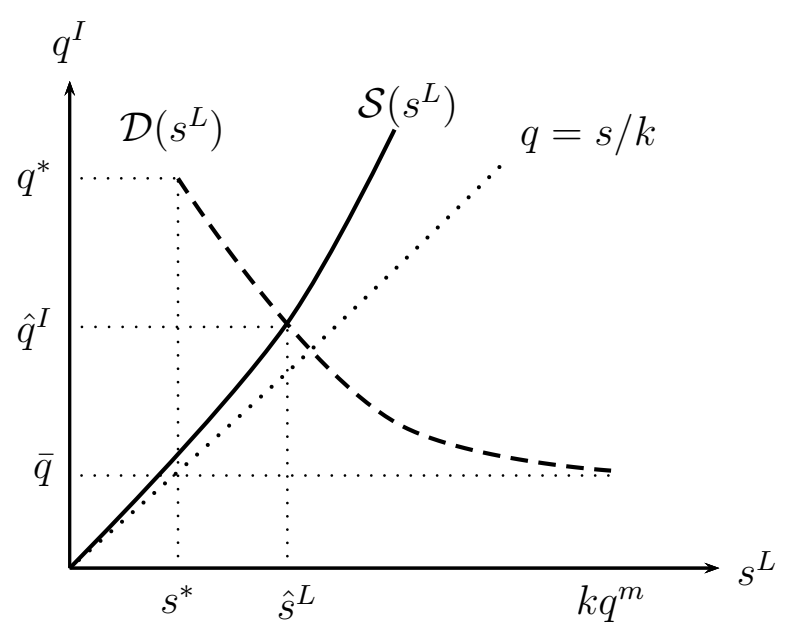

Figure 2: Equilibrium

the demand ultimately declines to $\bar{q}$, which gives the same consumption-utility as the outside option offered by the substitute. The demand schedule reaches $\bar{q}$ when the belief is optimistic enough, $s^{L} \geq k q^{m}$; then, after stopping, all potential seller types supply the static monopoly level that maximizes instantaneous profits. Hence, resource depletion does not affect post-stopping supplies, and the buyer does not require a compensation for the risk of supply disruption. Intuitively, when the seller is expected to be sufficiently large, the buyer does not demand a compensation for scarcity and will receive the same surplus flow under continuation as in the long-run from the outside option.

The supply schedule, denoted by $q^{I}=\mathcal{S}\left(s^{L}\right)$, identifies the maximum supply that the seller type $s^{L}$ is willing to offer to support continuation. As expected, the seller type and maximal supplies are positively correlated: the seller's opportunity costs of inducing stopping increase with the size of the stock that has to be sold in the limited time window of length $k$. Thus, with the stock size also increases the willingness to supply large amounts if such prevents stopping.

The area above the "demand" schedule and, simultaneously, below the "supply" schedule presents the potential outcomes for a stationary equilibrium. But, as we will see, for any $\left(s^{L}, q^{I}\right)$ strictly below the supply schedule, there are also seller types $s<s^{L}$ that will supply the required $q^{I}$, and thus the belief that the smallest type is $s^{L}$ is not Bayesian consistent. Thus, the equilibrium must be on the supply curve. For any point strictly above the demand curve, there exists a supply level $q<q^{I}$ for which the buyer prefers to continue, given the belief. Sellers will exploit this understanding of the buyer's 
incentive and offer supplies below $q^{I}$. Thus the equilibrium must be on the demand curve. The sections below provide the details for these arguments, the precise conditions under which a unique equilibrium exists, the comparative statics for the model's parameters, and an extension of the basic model to time-changing outside options.

\section{Equilibrium analysis}

\subsection{Supply: Seller's incentives}

The stock size is not observed by the buyer when the relationship continues but stopping forces the seller to reveal this information; after stopping, the game is over, and the only remaining issue for the seller is how to allocate the stock at hand for the known timeto-build period, after which the market for the resource dies out. Thus, in this sense, stopping puts the remaining resource to the market. If the stopping decision is made at $t$, the seller's optimal supply flow at each $\tau \in[t, t+k]$, which is the remaining time-window for sales, is

$$
q_{\tau}=\min \left\{s_{t} / k, q^{m}\right\}
$$

where $q^{m}=\operatorname{argmax}\{\pi(q)\}$ is the (static) monopoly supply in the absence of resource stock constraints. The strategy is simple: the post-stopping monopolist cannot do better than to sell flow $q^{m}$ but it may not have a stock large enough. If the stock falls short of $k q^{m}$, the best supply is $s_{t} / k$, exhausting the resource during the monopoly's remaining lifetime. If the stock is large enough so that $s_{t} / k \geqslant q^{m}$, there is no scarcity in the sense that post-stopping supply does not change with a larger holding; the seller will leave quantity $s_{t}-q^{m} k$ of the resource in the ground. ${ }^{14}$

Given the buyer's requirement for continuation $q^{I}$, the seller faces a simple opt-out problem: for how long to supply at least $q^{I}$, that is, for how long to implement continuation? Supplying $q_{t}<q^{I}$ triggers stopping and, through policy (1) over $[t, t+k]$, the stopping payoff is

$$
V_{t}^{I}=V^{I}\left(s_{t}\right)=\left\{\begin{array}{c}
k \pi\left(s_{t} / k\right) \text { if } s_{t}<k q^{m} \\
k \pi\left(q^{m}\right) \text { otherwise. }
\end{array}\right.
$$

As the stock declines, continuation becomes more costly to the seller because the

\footnotetext{
${ }^{14}$ Note that when there is scarcity, the monopoly's supply is socially optimal; however, the monopoly's threshold stock $q^{m} k$ for leaving resource in the ground is not socially optimal. Moreover, it should be noted that, when $s_{t}>k q^{m}$, stopping does not reveal fully the seller's stock level, only that there is at least $k q^{m}$. But, the payoff-relevant information is revealed.
} 
stopping value of the resource depends positively on the stock,

$$
V^{I \prime}\left(s_{t}\right)=\left\{\begin{array}{c}
\pi^{\prime}\left(s_{t} / k\right)>0 \text { if } s_{t}<k q^{m} \\
0 \text { otherwise }
\end{array}\right.
$$

At time $t=0$, each seller knows its initial stock $s_{0}$ and so can plan for opt-out time $T=T_{s_{0}}$ and also supply $q_{t} \geq q^{I}$ for all $t<T$ to implement continuation up to $T$. We can write the value of this program as

$$
V\left(s_{0}\right)=\max _{\left\{q_{t} \geq q^{I}, T\right\}} \int_{0}^{T} \pi\left(q_{t}\right) d t+V^{I}\left(s_{0}-Q_{T}\right),
$$

where $Q_{t}$ is the cumulative sum of the supplies at time $t$.

The seller's opt-out problem has an intuitive solution: the seller continues by offering the lowest possible supply $q^{I}$ as long as the price $p\left(q^{I}\right)$ from continuation sales exceeds the marginal decline in the stopping payoff, $V^{I \prime}\left(s_{t}\right)$, induced by depleting the stock at rate $q^{I}$. But since the decline in the stopping payoff is the marginal profit from selling the post-stopping quantity (see Fig. 3), the opt-out decision defines the marginal continuing type through the simple rule (5) in:

Lemma 1 For $p\left(q^{I}\right)>0$, the lowest continuing type, $s^{L}$ with $q^{I}=\mathcal{S}\left(s^{L}\right)$, is uniquely defined through the incentive constraint

$$
p\left(q^{I}\right)=\pi^{\prime}\left(\frac{s^{L}}{k}\right) .
$$

Moreover, $\mathcal{S}(0)=0, \mathcal{S}\left(s^{L}\right)>s^{L} / k$ for $s^{L}<k q^{m}$, and $\mathcal{S}\left(s^{L}\right)$ is continuous, and strictly increasing for $0 \leqslant s^{L}<k q^{m}$, and constant at $\mathcal{S}\left(s^{L}\right)=q^{u}$ for $s^{L} \geq k q^{m}$, where $q^{m}$ is the unconstrained monopoly supply that maximizes instant profits and $q^{u}$ is satiation supply, $p\left(q^{u}\right)=0 .{ }^{15}$ For all $\left(t, s_{t}\right)$ such that $\mathcal{S}\left(s_{t}\right) \geq q^{I}$, the privately optimal supply is $q_{t}=q^{I}$.

\subsection{Demand: consumer indifference}

The key step in the construction of the demand schedule is the description of the terms of trade that keeps the buyer indifferent between stopping the resource dependence and continuing. When contemplating stopping, the consumer does not yet know the seller's private information and thus cannot tell the supply that follows the stopping decision. But, understanding the seller's policy in (1), the buyer can form an expectation for the post-stopping surplus flow in $[t, t+k]$, using $\hat{u}(s / k)=u\left(\min \left\{s / k, q^{m}\right\}\right)$. Stopping with

\footnotetext{
${ }^{15}$ If the consumer's utility does not satisfy finite satiation, $q^{u}=\infty$, then $\mathcal{S}\left(s^{L}\right) \rightarrow \infty$ as $s^{L} \nearrow k q^{m}$.
} 


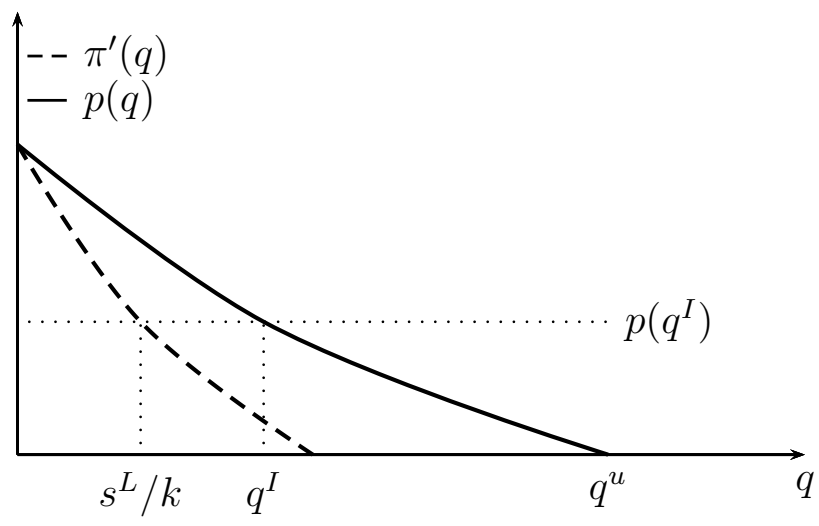

Figure 3: Demanded $q^{I}$ and the separating type $s^{L}$.

belief $s^{L}$, the total expected surplus for the post-investment phase, denoted by $\mathbb{E}\left[W^{I} \mid s^{L}\right]$, is the sum of the instantaneous surpluses in excess of the long-run surplus flow: ${ }^{16}$

$$
\mathbb{E}\left[W^{I} \mid s^{L}\right]=\int_{s^{L}}^{\infty} k[\hat{u}(s / k)-\bar{u}] d F\left(s ; s^{L}, \alpha\right) .
$$

This payoff measures the value of the outside option; it is independent of time and, for shorthand, we may write for the left-hand side $\mathbb{E} W^{I}=\mathbb{E}\left[W^{I} \mid s^{L}\right] .{ }^{17}$

Knowing what the consumer can expect from stopping, we have a basis for constructing the payoff from continuation. Specifically, we have conjectured that continuation is supported by a constant supply path which generates a payoff that is at least $\mathbb{E} W^{I}$. Let $q^{I}>0$ denote the conjectured constant supply that supports continuation and, for this path, let $\mathbb{E} W^{C}=\mathbb{E}\left[W \mid q^{I}, s^{L}\right]$ be the associated total surplus when the belief is $s^{L}$. We identify the cutoff supply $q^{I}$ through the indifference $\mathbb{E} W^{C}=\mathbb{E} W^{I}$.

Thus, consider a continuation consumption labeled $q^{I}$ over a short period $\varepsilon$, and the

\footnotetext{
${ }^{16}$ To be sure, in the Appendix, we derive this payoff as a limit of a traditional discounted surplus measure. As noted in Section 2.1, the payoff criterion here coincides with Dutta's (1991) strong long-run average payoff expression.

${ }^{17}$ Note that if the buyer's belief is that the seller has more stock than what will be supplied during the time to build, $s^{L}>k q^{m}$, then the stopping payoff is just $\mathbb{E} W^{I}=k\left[u\left(q^{m}\right)-\bar{u}\right]$, which is positive if the seller can offer surplus above the outside option. The payoff is also strictly increasing in dependence parameter $k$. This captures an element of waste in stopping when beliefs are very optimistic: for such beliefs, it is good for the buyer's payoff if the transition could be made longer through larger $k$. However, as we will see, such a situation is never relevant in equilibrium. In equilibrium, scarcity is expected, $s^{L}<k q^{m}$, and the consumer's perception of scarcity will increase with a longer period of dependence (Lemma 6 below).
} 
total payoff from time $t$ onwards, which equals the surplus generated over the interval $[t, t+\varepsilon]$, plus the payoff after that period. There is the probability $\varepsilon h$ with $h=\alpha q^{I}$ for the event that at time $t+\varepsilon$ the buyer will not receive $q^{I}$ but will learn that the seller is of a small type $s_{t} \in\left(s^{L}, s^{L}+\varepsilon q\right]$, in which case the future payoff becomes $k\left[\hat{u}\left(s^{L} / k\right)-\bar{u}\right]$. In the complement event, the buyer does not learn the seller's type, and continues with the same expected payoff, $\mathbb{E} W^{C}$. The expected surplus under continuation satisfies thus

$$
\mathbb{E} W^{C}=\varepsilon\left[u\left(q^{I}\right)-\bar{u}\right]+\varepsilon h k\left[\hat{u}\left(s^{L} / k\right)-\bar{u}\right]+(1-\varepsilon h) \mathbb{E} W^{C} .
$$

The last two terms contain a measure for the costs of delay. The items multiplied by $\varepsilon h$ denote the drop in the expected payoff in the situation where the seller turns out to be small: the expected continuation surplus minus the worst outcome surplus, $k\left[\hat{u}\left(s^{L} / k\right)-\bar{u}\right]$. We now want to identify $q=q^{I}$ that equates the continuation and stopping payoffs, $\mathbb{E} W^{C}=\mathbb{E} W^{I}$. When the indifference holds, the cost of delay can be expressed in a very useful way (as we prove in the Appendix):

Remark 1 (Cost of delay) Consider the limit $\varepsilon \rightarrow 0$. Then, if $\mathbb{E} W^{C}=\mathbb{E} W^{I}$, the following two measures for the cost of delay are equal:

$$
h\left(\mathbb{E}\left[W^{I} \mid s^{L}\right]-k\left[\hat{u}\left(s^{L} / k\right)-\bar{u}\right]\right)=q \mathbb{E}\left[\hat{u}^{\prime}\left(s_{t} / k\right) \mid s^{L}\right] .
$$

The left-hand side measures the cost of delay as the expected drop in payoff associated with learning that the seller is small. Intuitively, the right-hand side $q \mathbb{E}\left[\hat{u}^{\prime}(s / k) \mid s^{L}\right]$ is a measure of the expected scarcity, unavoidable when consumption continues at rate $q$ and the arrival of the alternative is postponed.

Combining (7)-(8) and the indifference $\mathbb{E} W^{C}=\mathbb{E} W^{I}$, gives

$$
\begin{aligned}
\mathbb{E} W^{I} & =\varepsilon[u(q)-\bar{u}]+\mathbb{E} W^{I}-\varepsilon q \mathbb{E}\left[\hat{u}^{\prime}(s / k) \mid s^{L}\right] \Rightarrow \\
u(q) & =\bar{u}+q \mathbb{E}\left[\hat{u}^{\prime}(s / k) \mid s^{L}\right] .
\end{aligned}
$$

This now defines the indifference-making supply $q=q^{I}$ which, after separating out the belief-dependent part, can be better seen from

$$
\begin{aligned}
u\left(q^{I}\right) & =\bar{u}+\lambda\left(s^{L}\right) q^{I} \\
\lambda\left(s^{L}\right) & =\mathbb{E}\left[\hat{u}^{\prime}(s / k) \mid s^{L}\right]=\int_{s^{L}}^{\infty} \hat{u}^{\prime}(s / k) d F\left(s ; s^{L}, \alpha\right) .
\end{aligned}
$$

Note that $\lambda=\lambda\left(s^{L}\right)$ is a number that depends, aside from the primitives, only on the buyer's beliefs as captured by $s^{L}$. Intuitively, the supply today should provide surplus 
$u(q)$ that is enough to cover (i) the substitute surplus, $\bar{u}$, that is lost irreversibly at this rate if the arrival of the substitute is postponed, and (ii) the expected scarcity cost $\lambda$ per unit of consumption $q$.

Through (11), we have a relationship between the demanded quantity for continuation and the belief, $q^{I}=\mathcal{D}\left(s^{L}\right)$; that is, the buyer's "demand" schedule depicted in Fig. 2 . For a formal statement, we first note how beliefs shape the expected scarcity cost:

Remark 2 Scarcity cost $\lambda\left(s^{L}\right)$ is a strictly decreasing function of $s^{L} \in\left(0, k q^{m}\right)$ :

$$
s^{L^{\prime}}>s^{L} \Rightarrow F\left(s, s^{L^{\prime}}, \alpha\right)>F\left(s, s^{L}, \alpha\right) \text { for all } s>s^{L} \Rightarrow \lambda\left(s^{L^{\prime}}\right)<\lambda\left(s^{L}\right)
$$

where $s^{L}<k q^{m}$. For $s^{L} \in\left[k q^{m}, \infty\right), \lambda\left(s^{L}\right)=0$.

Beliefs have an impact on the distribution of supply outcomes after stopping, provided the seller is expected to use all of its holdings at least in some outcomes (ensured by $\left.s^{L}<k q^{m}\right)$; otherwise, there is no perceived scarcity and thus $\lambda\left(s^{L}\right)=0$.

If beliefs are very pessimistic, the perceived scarcity cost may be larger than what the consumer can accept - it may not be possible to make the consumer indifferent. Define the largest scarcity cost that the consumer accepts, from (11), as

$$
\lambda^{*}=\max _{q}\left\{\frac{u(q)-\bar{u}}{q}\right\}=\frac{u\left(q^{*}\right)-\bar{u}}{q^{*}} .
$$

If $\lambda=\lambda^{*}$, it is possible to offer $q^{*}=\operatorname{argmax}\{(u(q)-\bar{u}) / q\}$ and make the buyer indifferent but the indifference breaks down if $\lambda$ is any larger; see Figure 4. Thus, for $\lambda\left(s^{L}\right)>\lambda^{*}$, it immediately follows that no continuation stage can exist, and the buyer immediately invests. On the other hand, if the belief implies that the seller's stock is so large that it will in all cases be left partially in the ground, $s^{L} \geq k q^{m}$, then there is no scarcity cost $\lambda=0$. In that case, continuation requires only a supply that provides the same surplus as the buyer's outside option, $q^{I}=\bar{q}=u^{-1}(\bar{u})$.

Assumption 1 For the most pessimistic belief $\left(s^{L}=0\right)$, the buyer will stop:

$$
\lambda(0)=\int_{0}^{\infty} \hat{u}^{\prime}(s / k) d F(s ; 0, \alpha)>\lambda^{*} .
$$

For sufficiently optimistic belief such that $s^{L} \geqslant k q^{m}$ (i.e., $\left.\lambda\left(s^{L}\right)=0\right)$, there exists continuation supply $q^{I}=\bar{q}=u^{-1}(\bar{u})<q^{m}$.

The assumption is not needed for Theorem 1 but, for discussion, it is natural to limit the parameter space $\Omega$, through Assumption 1, to focus on non-degenerate equilibrium 
outcomes. First, it states that beliefs need to be sufficiently optimistic to make the buyer's continuation possible. Second, it limits the strength of the buyer's outside option, so that compensating supply $\bar{q}=u^{-1}(\bar{u})$ exists, in case there is no expected scarcity, $\lambda=0$. Moreover, we limit the continuation supply to be less than the static monopoly supply $\bar{q}<q^{m} .{ }^{18}$

Condition (14) allows us to define the domain of the buyer's demand schedule:

Lemma 2 Given Assumption 1, there is a critical belief $s^{*} \in\left(0, k q^{m}\right)$ such that $\lambda\left(s^{*}\right)=$ $\lambda^{*}$ and $\mathcal{D}\left(s^{*}\right)=q^{*}$.

We can immediately see why such a critical belief must exist. By (14), sufficiently low expectation triggers stopping while belief $s^{L} \geqslant k q^{m}$ implies $\lambda=0$ and the feasibility of continuation. The expectation of scarcity, $\lambda\left(s^{L}\right)$, is continuously declining in belief so that $s^{L}=s^{*}$ solving $\lambda\left(s^{*}\right)=\lambda^{*}$ is unique. Note that $\lambda^{*}$ depends on $\bar{u}$, and that $\lambda\left(s^{L}\right)$ depends on $\alpha$ and $k$ so that $s^{*}$ depends on all parameters $\alpha, k, \bar{u}$.

Lemma 3 For all beliefs $s^{L}$ more optimistic than the critical belief $\left(s^{L} \geqslant s^{*}\right)$, the buyer's reservation demand $q^{I}=\mathcal{D}\left(s^{L}\right)$, defined through the indifference in (11), declines strictly in $s^{L}$ from $\mathcal{D}\left(s^{*}\right)=q^{*}$ until for $s^{L} \geqslant k q^{m}$ it takes value $\mathcal{D}\left(s^{L}\right)=\bar{q}=u^{-1}(\bar{u})$. For all beliefs $s^{L}<s^{*}$, no stationary supply can make the buyer to continue.

If (14) stated by Assumption 1 does not hold, then $s^{*}=0$. On the other hand, if, in Assumption 1, supply ensuring surplus $\bar{u}$ does not exist, the critical belief $s^{*}$ tends to infinity; the consumer invests immediately since the outside option dominates whatever the surplus is that the seller might offer.

\subsection{Equilibrium continuation and dynamics}

We can now characterize the determinants of the equilibrium resource relationship. From the analysis of the buyer's problem, we know that the buyer tolerates expected scarcity, as measured by $\mathbb{E}\left[\hat{u}^{\prime}\left(s_{t} / k\right) \mid s^{L}\right]$, up to $\lambda^{*}$, which is a given number defined by the buyer's primitive payoff expressions in (13). Continuation, then, requires that the buyer trusts the relationship enough, meaning a sufficiently large expected remaining stock, so that $\lambda\left(s^{L}\right)<\lambda^{*}$. Using the seller's incentives, the buyer can readily infer whether there can be enough trust in the relationship. There is a dichotomy based on the fundamentals that determines if there can be enough trust for continuation.

\footnotetext{
${ }^{18}$ This is to avoid equilibria where the scarcity considerations do no play a role; see the proof of Theorem 1 where we relax the assumption.
} 


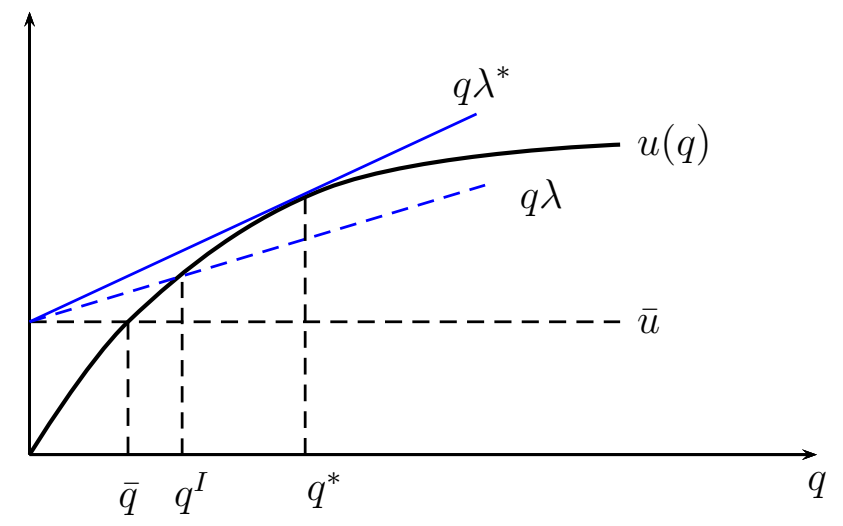

Figure 4: Determination of $q^{I}$ and $\lambda^{*}$

Proposition 1 Given Assumption 1, it holds for the stationary equilibrium resource relationship that

- If $\mathcal{S}\left(s^{*}\right) \leq q^{*}$, there is a unique continuation equilibrium that is ended by the informed party (seller) with probability one as time extends to infinity. That is, a unique pair of beliefs and supplies $\left(\hat{s}^{L}, \hat{q}^{I}\right)$ exists that satisfies seller's incentives (5) and buyer's indifference (11). Moreover, $\hat{s}^{L}<k q^{m}, \bar{q}<\hat{q}^{I} \leq q^{*}$.

- If $\mathcal{S}\left(s^{*}\right)>q^{*}$, there is no belief $s^{L}$ satisfying the continuation conditions. The relationship is ended by the uninformed party at $t=0$.

The dichotomy is thus the following. The smallest type that complies with the buyer's largest conceivable supply requirement has stock $s^{*}=\mathcal{S}^{-1}\left(q^{*}\right)$. This defines a pessimistic conjectural belief that allows the buyer to test whether the expected scarcity can in principle be less than what the buyer can tolerate. If $\mathcal{S}\left(s^{*}\right) \geqslant q^{*}$, which the buyer can verify from the seller's incentive constraint, seller type $s^{*}$ would be willing to go above $q^{*}$ for continuation; the true equilibrium belief is then more optimistic than $s^{*}$ and can be uniquely defined as well as the associated supply. Figure 2 depicts such a situation: looking at level $q^{*}$, the marginal supplying type $\mathcal{S}^{-1}\left(q^{*}\right)$ is larger than the buyer's equilibrium belief for smallest type that is willing to supply $q^{*}=\mathcal{D}\left(s^{*}\right)$. For this reason, and given the established properties of the buyer's demand and the seller's incentives, we can find the unique intersection of the two graphs.

Otherwise, the buyer's requirement and the sellers' incentives are incongruent, leading to immediate stopping. In Figure 2, this happens when the buyer's demand schedule 
shifts enough horizontally to the right so that the most pessimistic belief under which the buyer continues is higher than the marginal seller willing to implement continuation. Later, in Section 5, we introduce shocks to the options outside the resource relationship, so that the incentive incongruence can arise later in time than $t=0$.

Importantly, the continuation equilibrium features a time path for supplies where each privately informed type supplies the demanded $\hat{q}^{I}$ until the stock dwindles enough to reach $\hat{s}^{L}$, the seller's separation stock level. Each type thus separates at some point as $t \rightarrow \infty$ but, from the buyer's point of view, at random time. The separation is implemented through a supply shock; see Fig. 3 that shows how the continuation quantity differs from what the seller finds optimal to supply conditional on stopping. The size of the shock is public information as the buyer can infer the separating type and its post-stopping supply. We analyze next, among other substantial implications, how the supply shock depends on the fundamentals describing the relationship.

\section{Trust, dependence, and supply shocks}

We describe now how the equilibrium depends on: the buyer's primitive expectations with regards to the size of the stock, as measured by $\alpha$; the buyer's dependence on the seller, as measured by $k$; and the outside option $\bar{u}$. We consider changes in these fundamentals one at a time, and introduce them to the equilibrium relationships one by one. Consider first $\alpha$, and the buyer's perceived scarcity cost for given $\alpha$ and belief $s^{L}$ :

$$
\lambda\left(s^{L}, \alpha\right)=\int_{s^{L}}^{\infty} \hat{u}^{\prime}(s / k) d F\left(s ; s^{L}, \alpha\right) .
$$

Lemma 4 Scarcity cost $\lambda\left(s^{L}, \alpha\right)$ is increasing in $\alpha$ :

$$
\alpha^{\prime}>\alpha \Rightarrow F\left(s ; s^{L}, \alpha^{\prime}\right)<F\left(s ; s^{L}, \alpha\right) \text { for all } s>s^{L} \Rightarrow \lambda\left(s^{L}, \alpha^{\prime}\right)>\lambda\left(s^{L}, \alpha\right)
$$

where $s^{L}<k q^{m}$.

For larger $\alpha$, distribution $F$ becomes worse in the sense of stochastic dominance by the fact that $\alpha$ is a parameter of the exponential. Since $\hat{u}^{\prime}($.$) is a non-increasing function,$ value $\lambda\left(s^{L}, \alpha\right)$ increases.

We can now consider the effect of changes in $\alpha$ on the equilibrium; it enters the buyer's demand $q^{I}=\mathcal{D}\left(s^{L}, \alpha\right)$ that is defined through $u\left(q^{I}\right)=\bar{u}+\lambda\left(s^{L}, \alpha\right) q^{I}$ but it does not enter the seller's incentive constraint for continuation. Since larger $\alpha$ makes the distribution for types worse, the other part of the belief, that is $s^{L}$, must improve to keep the buyer indifferent. 
Lemma 5 The buyer's demand schedule $q^{I}=\mathcal{D}\left(s^{L}, \alpha\right)$, for $s^{L} \in\left[s^{*}, k q^{m}\right]$, shifts outwards (to the right) for increasing $\alpha$ :

$$
\alpha^{\prime}>\alpha \Rightarrow \mathcal{D}\left(s^{L}, \alpha^{\prime}\right)>\mathcal{D}\left(s^{L}, \alpha\right)
$$

and the critical belief $s^{*}$, defined Lemma 2, increases alongside. There is a maximal $\alpha^{*}$ that can support a stationary equilibrium, such that $q^{*}=\mathcal{S}\left(s^{*}\right)$.

Since the buyer's demand schedule shifts horizontally to the right with increased pessimism, the demanded supplies increase until no continuation in equilibrium can be supported. This is formalized below.

Proposition 2 (trust) There is a threshold $\alpha=\alpha^{*}>0$ such that for $\alpha=\alpha^{*}$ the unique stationary equilibrium supply is $\hat{q}^{I}=q^{*}$. For increased pessimism $\left(\alpha>\alpha^{*}\right)$, no continuation equilibrium exists. For increased optimism $\left(\alpha<\alpha^{*}\right)$, the continuation equilibrium exists; equilibrium supply $\hat{q}^{I}$ strictly decreases as $\alpha$ decreases and, moreover, $\hat{q}^{I} \rightarrow \bar{q}$ as $\alpha \rightarrow 0$.

The buyer places less trust in the relationship when $\alpha$ is increased, and therefore requires larger supplies (lower prices) for compensation. A sufficient increase in pessimism must lead to ending of the relationship. Strikingly, the increase in scarcity, as measured by $\alpha$, leads to larger supplies rather than smaller, in contrast with standard exhaustibleresource theory (see, Dasgupta and Heal, 1979). The difference is explained by elements in our setting that introduce caution on the consumer side, that is, the buyer's necessary dependence on the resource through the time-to-build period, and also by strategic interactions that allow bribing for continuation through generous supplies.

Proposition 3 (supply shock) An increase in the buyer-side caution through pessimism (larger $\alpha$ ) leads to a larger expected supply both before and after stopping, and a larger disruption when stopping.

An increase in the arrival rate for a small stock per unit of consumption, $\alpha$, shifts the buyer's demand schedule to the right in Lemma 5. When the buyer's demanded quantity for continuation increases and, consequently, consumption price $p\left(q^{I}\right)$ decreases, the marginal seller type willing to offer such terms of trade must increase. Since the marginal revenue falls faster than the price, the gap between continuation and stopping supplies must increase; see Fig. 3. In other words, the anticipated supply disruption increases with the buyer side caution, $\alpha$. 
An improvement in the buyer's outside option utility $\bar{u}$ has analogous implications on the equilibrium: the buyer's demand schedule for continuation $q^{I}=\mathcal{D}\left(s^{L}, \bar{u}\right)$ shifts to the right while the seller side incentives remain unaffected.

Proposition 4 There is a threshold $\bar{u}^{*}$ such that for $\bar{u}=\bar{u}^{*}$ the unique stationary equilibrium satisfies $\hat{q}^{I}=q^{*}$. For better substitutes, no continuation equilibrium exists. For worse substitutes (lower $\bar{u}$ ), the equilibrium supply $q^{I}$ decreases, approaching $q^{I}=0$ for $\bar{u}=0$. Moreover, given continuation, there is a larger supply disruption at stopping, the greater is $\bar{u}$

Time-to-build, as captured by $k$, measures the buyer's dependence on the seller but it also has direct implications for the seller's incentives. For the seller, the incentive to opt-out from continuation depends on $k$ since a longer time-to-build increases the value of stopping for a given stock level; separation schedule $q^{*}=\mathcal{S}\left(s^{L}\right)$ rotates right in Fig. 2 when $k$ is marginally increased. Intuitively, when $k$ extends to infinity, the upper bound for the seller's profits is approached because the resource stock is then effectively sold unit by unit at a price close to the maximum price $p(0)$.

The dependence period $k$ has an expected impact on the buyer's continuation demand; a longer time-to-build increases the perceived scarcity.

Lemma 6 Scarcity cost $\lambda\left(s^{L}, k\right)$ is increasing in $k$ : for any belief $s^{L}<k q^{m}$,

$$
k^{\prime}>k \Rightarrow \lambda\left(s^{L}, k^{\prime}\right)>\lambda\left(s^{L}, k\right) .
$$

For larger $k$, the buyer thus finds stopping more appealing, all else equal. So, distribution $F$ must become more favorable through an increase in belief $s^{L}$ to keep the indifference at given supply $q^{I}$, as defined through $u\left(q^{I}\right)=\bar{u}+\lambda\left(s^{L}, k\right) q^{I}$; the buyer's schedule $q^{I}=\mathcal{D}\left(s^{L}, k\right)$ shifts horizontally to the right with $k$ in Fig. 2. When combined with the change in the seller's incentives, we can unambiguously pin down the effect of the time-to-build period on the equilibrium:

Proposition 5 (dependence) There is cut-off $k^{*}$ such that for $k=k^{*}$ the unique stationary equilibrium satisfies $\hat{q}^{I}=q^{*}$. For a longer time-to-build, no continuation equilibrium exists. For $k<k^{*}$, the equilibrium supply $\hat{q}^{I}$ decreases as $k$ decreases, reaching the buyer's outside option supply $q^{I}=\bar{q}$ for $k=0$.

Interestingly, when the buyer's outside option becomes readily available $(k \rightarrow 0)$, the buyer's share of the resource surplus vanishes; the surplus from supplies $q^{I}=\bar{q}$ is the same as without the resource. Thus, the inability to adjust demand immediately is the source of the buyer's bargaining power, giving a share of the resource surplus. 


\section{Shocks to outside options}

We have seen that the relationship can end in two basic ways. First, the uninformed party may stop immediately. Second, the continuation is ended by the informed party who reveals its type at stopping. The separation of types is rooted in the dynamic change of the type space, leading to an equilibrium path with a random supply shock from the uninformed (buyer's) perspective, and full final exhaustion of the resource. However, stopping also by the uninformed party can happen on the equilibrium path, rather than only at time $t=0$, if outside options change during the game. Since the substantial implications for the equilibrium path are different depending on whether it is the uninformed or informed party who initiates the ending, we consider now a situation where the buyer's outside option may change at some future point in time.

From Proposition 4, we see that when the outside utility, $\bar{u}$, jumps from below to exceed $\bar{u}^{*}$, the uninformed party will stop at the time when the outside option changes, and there is a breakdown of the relationship. Assume now a constant hazard rate $x>0$ for the arrival of news about such an event. That is, we consider an initial state $A$ with $\bar{u}^{A}<\bar{u}^{*}$, but assume probability rate $x$ for the news that a transition will occur to a new state $B$ with $\bar{u}^{B}>\bar{u}^{*}$. The ultimate long-run substitute surplus is then $\bar{u}^{B}$ so that this becomes the benchmark relative to which we evaluate the expected surplus. The substitute surplus at time $s>t$ from the perspective of time $t$ when no news has arrived, is given by

$$
\begin{aligned}
& \mathbb{E}_{t} \bar{u}_{s}=\bar{u}^{A} \text { for } s<t+k \\
& \mathbb{E}_{t} \bar{u}_{s}=e^{-x(s-t-k)} \bar{u}^{A}+\left(1-e^{-x(s-t-k)}\right) \bar{u}^{B} \text { for } s \geq t+k .
\end{aligned}
$$

Here, by assumption, the better outside option has an arrival time that goes beyond the transition time of length $k$. Note that news about the arrival time may be released today. Thus, when using the substitute in state $A$, the buyer's expected loss from having to wait for state $B$ is

$$
\mathbb{E}_{t} \int_{0}^{\infty}\left[\bar{u}_{s}-\bar{u}^{B}\right] d s=\left(k+x^{-1}\right)\left(\bar{u}^{A}-\bar{u}^{B}\right) .
$$

Rewrite $W^{i}$ with $i=A, B$ for the stopping payoffs in the two states, now expressed as

$$
\begin{aligned}
\mathbb{E} W^{A} & =\int_{s^{L}}^{\infty} k\left[\hat{u}(s / k)-\bar{u}^{B}\right] d F(s ; \cdot)+x^{-1}\left(\bar{u}^{A}-\bar{u}^{B}\right), \\
\mathbb{E} W^{B} & =\int_{s^{L}}^{\infty} k\left[\hat{u}(s / k)-\bar{u}^{B}\right] d F(s ; \cdot) .
\end{aligned}
$$


Restated, the value of receiving information (announcement) that the improved substitute will arrive is

$$
\mathbb{E}_{t} W^{B}-\mathbb{E}_{t} W^{A}=\frac{1}{x}\left(\bar{u}^{B}-\bar{u}^{A}\right) .
$$

The announcement thus saves the visit to the inferior outside option $\bar{u}^{A}$. This formalization of shocks to outside options allows us to analyze the shocks as if they were unanticipated (see the Appendix for the proofs):

Proposition 6 If a new substitute for which no continuation equilibrium exists, $\bar{u}^{B}>$ $\bar{u}^{*}$ arrives at hazard rate $x$, while for the current substitute a stationary continuation equilibrium exists, $\bar{u}^{A}<\bar{u}^{*}$, then the equilibrium outcome is unaffected by the better substitute until it has become known.

The potential arrival of a new substitute does not change the buyer's trade off: resource depletion leads to an increased resource scarcity in expectations, and this needs to be compensated by an additional consumer surplus as before: $u\left(q^{I}\right)=\bar{u}^{A}+\lambda q^{I}$. The arrival of the new substitute also affects the seller's payoff but not the optimal opt-out time for each seller type.

We come to the substance lessons from the extension:

Proposition 7 For outside options $\bar{u}^{A}<\bar{u}^{*}<\bar{u}^{B}$, the ending of the resource relationship is characterized by a dichotomy: either (i) the seller's stock swindles before news on $\bar{u}^{B}$ arrive and the continuation path is followed by a supply disruption and full exhaustion, or (ii) the news arrive and the buyer stops the relationship, leading to a supply shock that is up (down) when the remaining stock is large (small). In expectations, some resource will be left unused at the arrival of the new substitute.

This extension allows the continuation path to end by stopping either by the informed or uninformed party. In both cases, from the buyer's point of view, the equilibrium stopping occurs at a random time. On the other hand, the seller has a privately known planned stopping time but faces uncertainty whether that time will be reached; when that time is not reached, the seller may have to leave some of its stock in the ground, depending on the resource availability at that time.

\section{Discussion}

We have made several modeling choices to make progress on a previously unexplored problem. 
There is a set of assumptions ensuring that the stationary equilibrium description is feasible. First, we imposed stationarity of beliefs ruling out dynamic signaling schemes that could potentially facilitate a faster separation of types. This extension could potentially be more natural in a setting where there are multiple buyers whose competition can lead to alternative belief structures (in the spirit of Nöldeke and Van Damme 1990 and Swinkels (1999)). In our current setting, we wanted to avoid outcomes where the buyer threatens with beliefs to achieve a better "screening" of the seller types; to the best of our knowledge, there are no well-developed candidates for refinements on beliefs that could be invoked in our setting (see Janssen and Roy (2002) for a discussion in a context for dynamic trading with price-taking agents; Ausubel et al. (2002) discuss the issue in bargaining settings). However, it is not clear whether a plausible equilibrium outcome should be much affected by events that are off-equilibrium; there can be noise in actions and external information that may affect the market values of the resource (in the spirit of Kremer and Skrzypacz (2005) and Daley and Green (2012)), and this can make conditioning of policies on intricate screening structures involving off-equilibrium beliefs impossible.

Second, keeping beliefs stationary in a sense that they do not depend on the full history of the game, a deviation from the exponential distribution for types would lead to non-stationarity of the strategies. We believe that this latter extension to relaxing stationarity has a higher priority - it is important to understand how relevant the exponential distribution is for the substance matter of this paper. Building on our working paper (available on request), we now shortly explain changes implied by a uniform prior for types. Thus, consider that the buyer's belief at $t=0$ is described by parameters $\left(\sigma_{0}, \theta_{0}\right)$ where $\sigma_{0}$ is the expected resource stock and $\theta_{0}$ is the spread of the belief such that there is a uniform support of seller types $\left[s_{0}^{L}, s_{0}^{H}\right]=\left[\sigma_{0}-\theta_{0}, \sigma_{0}+\theta_{0}\right]$. At some later date, the belief is $\left(\sigma_{t}, \theta_{t}\right)$ and the seller's privately know stock is $s_{t}$. With a uniform posterior, the measure of scarcity, that we have so far denoted by $\lambda\left(s^{L}\right)$, depends now on $\left(\sigma_{t}, \theta_{t}\right)$ and can be explicitly written as follows:

$$
\lambda\left(\sigma_{t}, \theta_{t}\right)=\frac{k}{2 \theta_{t}}\left[\hat{u}\left(\frac{\sigma_{t}+\theta_{t}}{k}\right)-\hat{u}\left(\frac{\sigma_{t}-\theta_{t}}{k}\right)\right] .
$$

When the degree of asymmetric information is sufficiently small, that is $\theta_{0}$ is low, it is possible to construct an equilibrium where all seller types supply the buyer's demanded quantity for continuation until the buyer decides to end the relationship. To see why the buyer must ultimately stop, note first that if no separation is expected, $\theta_{t}=\theta_{0}$ for all times; the buyer does not learn about the spread of types. The belief becomes a 
"moving box", with $d \sigma / d t=-q_{t}$ as the mean stock must decline with consumption. But, then, by (16), the scarcity cost keeps on increasing with consumption, so that the buyer's indifference requirement must change over time as well.

On the other hand, a large informational asymmetry, $\theta_{0}$ above a certain threshold, leads to expected separation in equilibrium. More precisely, the buyer's equilibrium beliefs describe a truncation function for the type distribution, as the buyer can rule out seller types that should have triggered a supply shock given the publicly observed history of the interaction. Thus, the beliefs about the seller's size drift up at some rate which, however, generally differs from the drift down due the expected scarcity increase due to the cumulated consumption. The large asymmetry then leads to beliefs updating similar to the model presented in this manuscript.

In conclusion, the model with a non-stationary solution leads to similar quantitative conclusions: the consumer requires a compensation for continuing the relationship which is ended by a supply shock when the ending is initiated by the informed party. The economic reasoning for the sellers' late reporting of their types is exactly the same as in the exponential model. The conclusions regarding "trust", "dependence", and "supply shocks", delivered by the exponential model, seem qualitatively robust.

Our model departs by construction from the strand of literature that followed Akerlof (1970) and Spence (1973) to study the dynamics of hidden information: in this literature, when there are no informational asymmetries, one side of the market takes the full surplus, which is natural since the focus is on information-driven changes in surpluses and thus in efficiency. Our model preserves a non-trivial division surplus when there is full information about the stock size. This is best illustrated by the uniform distribution case. When $\theta_{0} \rightarrow 0$ in (16), the buyer knows the stock size precisely $s=\sigma$, and, under scarcity $s<k q^{m}$, the continuation supply is given by

$$
u(q)=\bar{u}+q u^{\prime}(s / k)
$$

This condition pins down the unique Markov-perfect equilibrium of the symmetric information case (Gerlagh and Liski, 2011). ${ }^{19}$ This illustration shows that the core of our model is a bargaining situation where inefficiencies are preserved even without hidden information. The distortions arise from the fact that transfers are market-based; without

\footnotetext{
${ }^{19}$ Unfortunately, the stationary equilibrium with exponential distribution of types is not appropriate for considering the full information limit because one parameter governs both the dispersion and the mean of the seller types, and therefore the full information limit is also the one where the scarcity vanishes.
} 
this assumption the parties could directly bargain about the division of the resource value with direct transfers (as, for example, in Schweinzer 2010). We have characterized the full information distortions in Gerlagh and Liski (2011).

One contribution of the current paper is that it can be either the bargaining or hidden information that shapes the equilibrium outcome. The bargaining outcome dominates when the buyer stops the relationship due to a good outside option, and therefore does not enter the dynamic signaling game at all (or may leave it after an improvement in the outside option). With full information, the buyer must ultimately stop when the seller's observable stock sufficiently declines; thus, bargaining leads to a final breakdown because of the stock depletion. Hidden information shapes the equilibrium when there is a bargaining outcome for continuation, and by the assumptions made on stationarity, hidden information always delegates the ending decision to the informed party. It is obvious that these properties arise in an fundamental way from the physical state of the problem that is endogenously developing in the equilibrium.

Our results can be linked to Hörner and Kamien (2004) who show that a resource monopsony facing price-taking and forward-looking sellers is conceptually equivalent to a durable-good monopoly facing price-taking and forward-looking buyers. In their paper, the Coase conjecture (Coase, 1972) arises since the low-cost resource sellers can wait for the high-cost seller to enter the market; in the undiscounted limit, the buyer's market power vanishes at the twinkling of an eye, as expressed by Coase for the durable-good monopoly. It is essential for the conjecture that the resource sellers have heterogenous costs of supplying, similarly as it is essential for the original durable-good monopoly that the consumers are heterogenous. In our model, there is no cost of extraction but the outside option has a similar role: there is a zero-cost finite resource, and a highercost substitute-resource that is infinite. Now, the Coase conjecture says that without discounting the buyer should receive no surplus from the resource; the seller should price the resource supplies at the cost of the substitute. Our equilibrium achieves this limit when the adjustment delay $k$ vanishes; the buyer receives the long-run payoff during the resource consumption period, and thus no resource surplus (Proposition 5). Since the seller takes the full surplus, the equilibrium outcome is also efficient, irrespective of the private information. The time-to-build period for the substitute is thus an additional feature of the cost structure that, when positive, leaves some surplus also for the buyer and is therefore the source of distortions in our setting.

We have eliminated discounting from the analysis for tractability, building on Dutta (1991) for the appropriate and intuitive tools that are applicable since the long-run state 
of the game is "absorbing". The assumption of no discounting ensures that there is an equilibrium characterized by the buyer's indifference between continuation and stopping; this may no longer hold when there is discounting. Intuitively, when the resource is very large, the end-game that has been the focus of this paper is far in the future and, by positive discounting, the seller gives more weight to current revenues leading to supplies closer to static monopoly supplies and potentially exceeding the buyer's demand for continuation. Thus, both parties can prefer continuation, which changes the nature of the analysis. Clearly, positive discounting is also important for descriptive realism.

We have also made very stark assumptions on the buyer's outside options, capturing the notion of a substitute for the resource. Descriptive realism can be added by considering a more gradual investment process, allowing the resource to compete with the substitute, or adding uncertainty to the transition period. We have analyzed such extensions for the symmetric information case (Gerlagh and Liski, 2011). The general conclusion from that analysis is that as long as the buyer's decision can irreversibly destroy part of the seller's surplus, there is something to be gained by offering part of that surplus to the buyer; this makes core dynamics of the current results robust to extensions mentioned. Note that the extensions modify the description of the post-stopping stage where the game is over; they seem largely inconsequential for the issues of hidden information considered in the current paper.

One final restrictive assumption that facilitated smooth analysis is the strict concavity of the buyer's surplus, $u(q)$. The assumption is very restrictive since, for example, it rules out linear demand. However, the construction of the equilibrium does not depend on concavity. For example, looking at Fig. 4, highest scarcity cost $\lambda^{*}$ that the buyer can tolerate can be found for any continuous and bounded surplus function. Nevertheless, uniqueness of the stationary equilibrium and monotonicity of the equilibrium with respect to parameter changes can depend on concavity. ${ }^{20}$

\section{Concluding remarks}

We started with an illustration from the past, the Chilean nitrate monopoly, which was ended by the buyer side action. Let us now close the plot by discussing other markets where it may be the seller side that initiates the transition to the alternative supplies.

\footnotetext{
${ }^{20}$ But even these properties can be restored by changing the distributional assumptions; for example, with a uniform type distribution, the concavity of $u(q)$ can be relaxed with the cost of losing stationarity. This version of the model is available on request.
} 
Conventional oil can be defined as the cheapest-to-extract oil reserve in the hands of a few core OPEC countries. ${ }^{21}$ It is this low-cost but finite reserve with concentrated ownership and inelastic short-run demand that is the exhaustible resource of interest for the issues raised in this paper; the rest of production can be seen as part of the substitute fuel production, including costly conventional oil sources, nonconventional oils, biofuels, and alternative energy sources. The industry experts estimates of the remaining viable core-oil stocks vary widely, which is a precondition for the equilibrium where the supply disruption is a possibility. ${ }^{22}$ Moreover, we have observed increasing supplies from such core sources over time, although the stocks are undisputedly declining.

Another exhaustible resource with concentrated ownership is phosporus that is mostly obtained from mined phoshate to produce fertilizers together with other mineral nutrients. Unlike in the case of Chilean nitrate or fossil fuels, the substitutes for such a basic mineral nutrient is yet to be discovered. It has only three major producers: United States (Florida), China, and Morocco/Western Sahara. Production in the US is dwindling and China does not export, leaving Morocco/Western Sahara as the main supplier in the world market. It has been estimated that the currently available resource stocks maybe depleted during the next 50-100 years; however, the estimates of the overall reserves vary considerably (Cordell et al. 2009; Keyzer 2010).

\section{Appendix}

\section{The buyer's strong long-run average payoff}

Here we derive the buyer's stopping payoff in (6) as a limit of a discounted payoff. Consider the following expected discounted payoff from stopping

$$
\mathbb{E}\left[U^{I} \mid s^{L}\right]=\int_{s^{L}}^{\infty} \int_{0}^{k}[\hat{u}(s / k)] e^{-\rho \tau} d \tau d F\left(s ; s^{L}, \alpha\right)+e^{-\rho k} \frac{1}{\rho} \bar{u}
$$

where $\rho>0$ is the discount rate. Define

$$
\mathbb{E}\left[W^{I} \mid s^{L}\right]=\mathbb{E}\left[U^{I} \mid s^{L}\right]-\frac{1}{\rho} \bar{u} .
$$

\footnotetext{
${ }^{21}$ See the " 2007 Medium-Term Oil Market Report" published by the International Energy Agency for estimates of the Core OPEC reserves. The Saudi share of the Core OPEC stocks is expected to increase over time.

${ }^{22}$ The estimates and concerns are extensively entertained in a popular book by Simmons (2005); see also the Hirsch Report (prepared for the U.S. Department of Energy, 2005).
} 
Letting $\rho \rightarrow 0$, gives the buyer's nondiscounted payoff (6) in the text. This is the strong long-run average payoff, as defined by Dutta (1991). The conditions stated in Dutta (1991) for this payoff criterion to identify the appropriate policies for the undiscounted limit are trivially satisfied in our setting.

\section{Proof of Remark 1}

Exploiting the exponential distribution's properties, such as $f_{s}\left(s ; s^{L}, \alpha\right)=-\alpha f\left(s ; s^{L}, \alpha\right)$, and $f\left(s^{L} ; s^{L}, \alpha\right)=\alpha$, we find that

$$
\begin{aligned}
\mathbb{E}\left[\hat{u}^{\prime}(s / k) \mid s^{L}\right] & =\int_{s^{L}}^{\infty} \hat{u}^{\prime}(s / k) f(s ; \cdot) d s \\
& =-\int_{s^{L}}^{\infty} k[\hat{u}(s / k)-\bar{u}] f_{s}(s ; \cdot) d s+[k[\hat{u}(s / k)-\bar{u}] f(s ; \cdot)]_{s^{L}}^{\infty} \\
& =\alpha\left\{\mathbb{E}\left[W^{I} \mid s^{L}\right]-k\left[\hat{u}\left(s^{L} / k\right)-\bar{u}\right]\right\} .
\end{aligned}
$$

Now, when we substitute $h=\alpha q$, we have (8). Q.E.D.

\section{Proof of Remark 2}

Scarcity cost is defined as

$$
\lambda\left(s^{L}\right)=\int_{s^{L}}^{\infty} \hat{u}^{\prime}(s / k) d F\left(s ; s^{L}, \alpha\right) .
$$

Increasing $s^{L}$ does not affect the utility but only the distribution. The distribution with higher $s^{L}$ stochastically dominates a one with lower $s^{L}$ :

$$
s^{L^{\prime}}>s^{L} \Rightarrow F\left(s ; s^{L^{\prime}}, \alpha\right)>F\left(s ; s^{L}, \alpha\right) .
$$

Since $\hat{u}^{\prime}(s / k)$ is a decreasing function, it follows that $\lambda\left(s^{L^{\prime}}\right)<\lambda\left(s^{L}\right)$, under the conditions stated. Q.E.D.

\section{Proof of Lemma 2}

In text. Q.E.D.

\section{Proof of Lemma 3}

Consider

$$
u(q)=\bar{u}+q \lambda
$$


for a given $\lambda<\lambda^{*}$. Differentiating with respect to $q$ and $\lambda$, yields

$$
\begin{aligned}
\hat{u}^{\prime}(q) d q & =\lambda d q+q d \lambda, \text { or } \\
\frac{d q}{d \lambda} & =\frac{q}{\hat{u}^{\prime}(q)-\lambda} .
\end{aligned}
$$

We obtain $d q / d \lambda>0$ since, by definition of $\lambda^{*}$, we have $\hat{u}^{\prime}(q)-\lambda>0$. Now, $\lambda$ is continuously decreasing in $s^{L}$ (by Remark 2), implying that the reservation supply $q^{I}$ is continuously increasing in $s^{L}$ whenever such a supply satisfying $u(q)=\bar{u}+q \lambda$ exists. By the same continuity argument and Lemma 2 such $q^{I}$ can exist only when $s^{L} \geqslant s^{*}$. For $s^{L} \geqslant k q^{m}, \lambda=0$ and $q=\bar{q}$, where $\bar{q}$ exists by Assumption 1 .

\section{Proof of Lemma 1}

We rewrite the value of the seller's program,

$$
\begin{gathered}
V\left(s_{0}\right)=\max _{\left\{q_{t} \geqslant \bar{q}, T\right\}} \int_{0}^{T} \pi\left(q_{t}\right) d t+V^{I}\left(s_{0}-Q_{T}\right) \\
\Rightarrow \\
V\left(s_{0}\right)=\max _{T}\left\{p\left(q^{I}\right) q^{I} T+V^{I}\left(s_{0}-q^{I} T\right)\right\},
\end{gathered}
$$

where the last line uses the fact that by keeping supplies at the buyer's reservation level, $q_{t}=q^{I}$, the seller receives the reservation price for continuation for all units in its reserve, until stopping at $T$.

The first-order condition for $T>0, p\left(q^{I}\right)>0$ is

$$
p\left(q^{I}\right)=V^{I \prime}\left(s_{0}-q^{I} T\right)=V^{I \prime}\left(s_{T}\right)
$$

where $V^{I \prime}\left(s_{T}\right)$ is defined in (3). Noting that the seller at time $T$ is the smallest seller type, so we can substitute $s_{T}=s^{L}$ and obtain for $p\left(q^{I}\right)>0$

$$
p\left(q^{I}\right)=\pi^{\prime}\left(\frac{s^{L}}{k}\right)
$$

which is a relationship between the demanded quantity $q^{I}$ and the minimal stock level for the seller's continuation. Since $\pi^{\prime}\left(\frac{s^{L}}{k}\right)$ strictly increases as $s^{L}$ declines, the equation uniquely defines the smallest continuing resource owner. We denoted this supplier as a function of the smallest seller type $q^{I}=\mathcal{S}\left(s^{L}\right)$.

For the rest of the properties, note that utility function $U(q)$ is twice continuously differentiable. Since then $p(q) \rightarrow \pi^{\prime}(q)$ as $q \rightarrow 0$, we have $\mathcal{S}(0)=0$. On the other hand, for $s^{L} / k<q^{m}$, since $\pi^{\prime}\left(s^{L}\right)<p\left(s^{L} / k\right)$, it follows from (18) that $\mathcal{S}\left(s^{L}\right)>s^{L} / k$. As $\pi^{\prime}\left(s_{L}\right)<0$, the seller will never stop if $s_{L}>k q^{m}$. It follows that $\mathcal{S}\left(s^{L}\right)=q^{u}$ for all $s^{L} \geq k q^{m}$, with $p\left(q^{u}\right)=0$. Q.E.D. 


\section{Proof of Proposition 1}

If we can find a pair $\left(\hat{s}^{L}, \hat{q}^{I}\right)$ such that $\mathcal{S}\left(\hat{s}^{L}\right)=\mathcal{D}\left(\hat{s}^{L}\right)=\hat{q}^{I}$, then, by construction, the buyer's (weak) best response is to continue given belief $\hat{s}^{L}$, and the seller type $s>\hat{s}^{L}$ (strict) best response is to supply $\hat{q}^{I}$. If such a pair does not exist, then there is no stationary continuation equilibrium. We consider the two conditions, $\mathcal{S}\left(s^{*}\right) \leq q^{*}$ and $\mathcal{S}\left(s^{*}\right)>q^{*}$, in the Proposition separately; by Assumption 1 and Lemma 2, critical belief $s^{*}>0$ dividing the two cases exists. For shorthand, we denote $s^{m}=k q^{m}$ in this proof.

\section{Continuation condition in Proposition 1:}

$$
\mathcal{S}\left(s^{*}\right) \leq q^{*}
$$

Thus,

$$
\mathcal{D}\left(s^{*}\right)=q^{*} \geqslant \mathcal{S}\left(s^{*}\right)
$$

so that "demand" exceeds "supply" for $s^{L}=s^{*}$ (type $s^{*}$ will not supply $q^{*}$ ). Lemma 3 states $\bar{q}=\mathcal{D}\left(s^{m}\right)$, and by Assumption $1, \bar{q}<q^{m}<q^{u}$ so

$$
\mathcal{D}\left(s^{m}\right)=\bar{q}<q^{u}=\mathcal{S}\left(s^{m}\right) .
$$

Thus, "supply " exceeds "demand" for $s^{L}=s^{m}$ (type $s^{m}$ could supply more than the required, $\bar{q}$, for continuation).

Since, by Lemma 3, the buyer's requirement $\mathcal{D}\left(s^{L}\right)$ is continuous and declining in $s^{L}$ from $q^{*}$ to $\bar{q}$, where the lower-end is reached when $s^{L}=s^{m}=k q^{m}$, it follows by (19) and (21) that the schedules $\mathcal{S}$ and $\mathcal{D}$ must intersect. The intersection is unique by Lemmas 1 and 3, defining the unique pair $\left(\hat{s}^{L}, \hat{q}^{I}\right)$ such that $\mathcal{S}\left(\hat{s}^{L}\right)=\mathcal{D}\left(\hat{s}^{L}\right)=\hat{q}^{I}$.

Finally, the small seller, type $\hat{s}^{L}$, triggering stopping arrives at rate $\alpha \hat{q}^{I}>0$ per unit of time. Thus, stopping occurs with probability one as $t \rightarrow \infty$.

\section{Stopping assumption in Proposition 1:}

$$
\mathcal{S}\left(s^{*}\right)>q^{*}
$$

so that

$$
\mathcal{S}\left(s^{*}\right)>\mathcal{D}\left(s^{*}\right)=q^{*}
$$

By the arguments from the first part of the proof, (22) rules out an intersection of $\mathcal{S}$ and $\mathcal{D}$. Note that the seller's continuation profit increases when supplies decrease below $\mathcal{S}(s)$ : all seller types $s^{L} \geqslant s^{*}$ can supply $q^{*}$ for continuation. However, this same argument holds for all types in $\left[\mathcal{S}^{-1}\left(q^{*}\right), s^{*}\right)$, where $\mathcal{S}^{-1}\left(q^{*}\right)<s^{*}$ by assumption. Thus, consistent belief implies $\lambda>\lambda^{*}$ for which continuation is not possible by the definition of $\lambda^{*}$ in (13). No stationary equilibrium can exist. Q.E.D. 


\section{Proof of Theorem 1}

The proof follows from the proof of Proposition 1, after we relax Assumption 1 which is not required in the Theorem.

If (14) stated by Assumption 1 does not hold, then $\mathcal{S}\left(s^{*}\right)=\mathcal{S}(0)=0$, by Lemma 1 , and the continuation condition in Proposition 1 is satisfied.

If $\bar{q}>q^{m}$, Assumption 1 is again violated. It implies that the buyer's outside utility flow exceeds what the monopoly after stopping can maximally offer, $\bar{u}>\hat{u}\left(q^{m}\right)$. The equilibrium degenerates as the buyer will not accept any expected scarcity. The equilibrium belief is $\hat{s}^{L}>k q^{m}$ (and $\lambda=0$ ); a seller with sufficient stock, $\mathcal{S}\left(q^{m}\right)>k q^{m}$, will supply $\hat{q}^{I}=\bar{q}=\hat{u}^{-1}(\bar{u})$, until its stock declines to level $k q^{m}$. Q.E.D.

\section{Proof of Proposition 2}

Restating (13),

$$
\lambda^{*}=\max _{q}\left\{\frac{u(q)-\bar{u}}{q}\right\}=\frac{u\left(q^{*}\right)-\bar{u}}{q^{*}}
$$

we can see that $\lambda^{*}$ is a positive constant, defined by the primitive payoff functions. Thus, through $\lambda^{*}=\lambda\left(s^{*}, \alpha\right)$, critical belief $s^{*}$ is a continuous and increasing function of $\alpha$, as in the proof of Lemma 5. Lemma 2 shows that such $s^{*} \in\left(0, k q^{m}\right)$ exists. Assumption 1 in Lemma 2 ensures that $s^{*}<k q^{m}$, for any given $\alpha<\infty$. Assumption 1 in Lemma 2 puts an implicit lower bound on $\alpha$ so that $\lambda^{*}<\lambda(0, \alpha)$ holds. This lower bound can be

relaxed as we explain shortly; for the time being, assume that $\alpha$ is above this lower bound so that $\lambda^{*}=\lambda\left(s^{*}, \alpha\right)$ holds for $s^{*}>0$. Denote $s^{*}=s^{*}(\alpha)$ such that $\lambda^{*}=\lambda\left(s^{*}(\alpha), \alpha\right)$.

We can now restate the condition for stopping in Proposition 1 as follows:

$$
\mathcal{S}\left(s^{*}(\alpha)\right)>q^{*} \Leftrightarrow \alpha>\alpha^{*}
$$

This proves Proposition 2, excluding the case where $\alpha$ is so low that Assumption 1 is violated, and $\lambda^{*}<\lambda(0, \alpha)$. For all $\alpha$ so low, we have $s^{*}=0$. However, the indifferencemaking supply is still determined by $u\left(q^{I}\right)=\bar{u}+q^{I} \lambda(0, \alpha)$, and therefore as $\alpha \rightarrow 0$, $q^{I} \rightarrow \bar{q}$

\section{Proof of Lemma 4}

Parameter $\alpha$ affects only the distribution and not the payoff function. The lemma states that $\alpha^{\prime}>\alpha \Rightarrow F\left(s ; s^{L}, \alpha^{\prime}\right)<F\left(s ; s^{L}, \alpha\right)$ for all $s>s^{L}$ (for $s \leqslant s^{L}$, the CDF is zero for all 
$\alpha$ ). The distribution is exponential, and $\alpha>0$ is the parameter of that distribution; hence the conclusion. Since the payoff function is non-increasing in the stochastic variable, the expected value increases in $\alpha$. The lemma requires $k q^{m}>s^{L}$; otherwise $\lambda=0$, for all $\alpha>0$ (the seller is expected to have more stock than what will be supplied). Q.E.D.

\section{Proof of Lemma 5}

From Remark 2, $\lambda\left(s^{L}, \alpha\right)$ is strictly increasing in $s^{L}$ given $\alpha$, and, from Lemma 4, we see that $\lambda\left(s^{L}, \alpha\right)$ is strictly decreasing in $\alpha$ given $s^{L}$. Differentiating $\hat{u}\left(q^{I}\right)=\bar{u}+q^{I} \lambda\left(s^{L}, \alpha\right)$ with respect to $s^{L}$ and $\alpha$ leads to $d s^{L} / d \alpha=-\lambda_{\alpha}\left(s^{L}, \alpha\right) / \lambda_{s^{L}}\left(s^{L}, \alpha\right)>0$.

From Proposition 1, an equilibrium exists if and only if $\mathcal{S}\left(s^{*}\right) \leq q^{*}$. Note that $q^{*}$ is a constant defined by (13), consistent with scarcity cost $\lambda^{*}$. Thus, for each $\alpha, \lambda\left(s^{*}, \alpha\right)=\lambda^{*}$ defines the critical belief $s^{*}$. Since $d s^{*} / d \alpha>0$, there is $\alpha^{*}>0$ such that $\mathcal{S}\left(s^{*}\right)=q^{*}$, and $\mathcal{S}\left(s^{*}\right)>q^{*}$ for $\alpha>\alpha^{*}$. Q.E.D.

\section{Proof of Proposition 3}

An in increase in $\alpha$ shifts the buyer's demand schedule to the right in Lemma 5 . Then, in view of Fig. 2, the separating type increases along $\mathcal{S}^{-1}\left(q^{I}\right)$ which is less than that indicated by the diagonal line $s=k q$; this follows directly from the monotonicity of the separation condition, defined through (5). Thus, increased pessimism leads to a larger expected supply disruption. Q.E.D.

\section{Proof of Proposition 4}

The buyer's demand for continuation, $q^{I}=\mathcal{D}\left(s^{L}, \bar{u}\right)$, experiences a qualitatively similar change as in Lemma 5 but now $\alpha$ is replaced by $\bar{u}$. The analysis of marginal changes is similar as in Proposition 2, and thus omitted. However, the global analysis is different: when $\bar{u}>\hat{u}\left(q^{m}\right)$, Assumption 1 is violated. That assumption was made to ensure that the main text can focus on an interior equilibrium but dropping Assumption 1 does not conceptually alter the equilibrium. Given $\bar{u}>\hat{u}\left(q^{m}\right)$, the equilibrium belief is $\hat{s}^{L}>k q^{m}$ (and $\lambda=0$ ); a seller with sufficient stock, $\mathcal{S}\left(k q^{m}\right)<q^{m}$, will supply $\hat{q}^{I}=\bar{q}=\hat{u}^{-1}(\bar{u})$, until its stock declines to level $k q^{m}$. Q.E.D. 


\section{Proof of Lemma 6}

For all $s^{L} \leq s$, we have $d F\left(s ; s^{L}, \alpha\right)>0$. Moreover, for any $s^{L}<k q^{m}$, we have $\hat{u}^{\prime \prime}(s / k)<$ 0. Thus,

$$
\frac{d}{d k} \lambda\left(s^{L}, k\right)=\int_{s^{L}}^{\infty} \frac{-s}{k^{2}} \hat{u}^{\prime \prime}(s / k) d F\left(s ; s^{L}, \alpha\right)>0
$$

Q.E.D.

\section{Proof of Proposition 5}

By definition in (13), $\lambda^{*}$ is independent of $k . \lambda\left(s^{L}, k\right)$ is increasing in $k$; from the proof of Lemma 6 , we can see that $\lambda\left(s^{L}, k\right)$ continuous and differentiable in $k>0$. Moreover, by Remark $2, \lambda\left(s^{L}, k\right)$ is decreasing (and differentiable) in $s^{L}$. Thus, $\lambda^{*}=\lambda\left(s^{L}, k\right)$ defines the critical belief $s^{L}=s^{*}$ as an increasing function of $k$ :

$$
\lambda^{*}=\lambda\left(s^{*}, k\right) \Rightarrow s^{*}=s^{*}(k), \frac{d s^{*}}{d k}=-\frac{\lambda_{k}\left(s^{*}, k\right)}{\lambda_{s}\left(s^{*}, k\right)}>0
$$

Now, in Proposition 1, the condition for stopping can be stated:

$$
\mathcal{S}\left(s^{*}(k)\right)>q^{*} \Leftrightarrow k>k^{*}
$$

where $k^{*}<\infty$ since $\mathcal{S}\left(q^{*}\right)$ is bounded. When $k \rightarrow 0$, we have $\mathbb{E} W^{I} \rightarrow 0$ from (6). Then, $W=\mathbb{E} W^{I} \Rightarrow u(q)=\bar{u} \Rightarrow \hat{q}^{I}=\bar{q}$. Q.E.D.

\section{Proof of Proposition 6}

We first reconstruct the buyer's indifference for the outside option state $A$. Consider a short time period of length $\varepsilon$. The buyer's payoff relative to the benchmark $\bar{u}^{B}$, after continuation consists of the consumer surplus above the long-run surplus, $u(q)-\bar{u}^{B}$. As before, there is a probability $\varepsilon h$ that the seller announces to be the smallest type $s^{L}$, after which the payoff outcome is $\hat{u}\left(s^{L} / k\right)-\bar{u}^{B}$ for $k$ units of time, and the expected postresource payoff equals $x^{-1}\left(\bar{u}^{A}-\bar{u}^{B}\right)<0$ thereafter. In addition, there is probability $\varepsilon x$ that the future arrival time of the better substitute is announced. In that case, the buyer will immediately invest after $\varepsilon$ time. If the buyer decides to invest because information on the new substitute has arrived, its expected cumulative payoff during the dependence stage will have decreased by $\varepsilon \lambda q=\varepsilon q k \mathbb{E}\left[\hat{u}^{\prime}(s / k) \mid s^{L}\right]$ units, while its post dependence expected surplus will have increased by $x^{-1}\left(\bar{u}^{B}-\bar{u}^{A}\right)$. The economy remains in the same 
stationary state with remaining probability $(1-\varepsilon h-\varepsilon x)$. Collecting these items, the analog of the continuation surplus (7) can now be stated:

$$
\begin{gathered}
\mathbb{E} W=\varepsilon\left[u(q)-\bar{u}^{A}\right]+\varepsilon h k\left[\hat{u}\left(s^{L} / k\right)-\bar{u}^{A}\right]-\varepsilon h x^{-1}\left(\bar{u}^{B}-\bar{u}^{A}\right) \\
+\varepsilon x\left(\mathbb{E} W^{B}-\varepsilon \lambda q\right)+(1-\varepsilon h-\varepsilon x) \mathbb{E} W .
\end{gathered}
$$

The buyer is indifferent between continuation and stopping, so we can we substitute $\mathbb{E} W=\mathbb{E} W^{A}$. We get (noticing that the term $\varepsilon^{2} x \lambda q$ cancels for $\varepsilon$ small):

$$
\begin{gathered}
0=\varepsilon\left[u(q)-\bar{u}^{A}\right]+\varepsilon h k\left[\hat{u}\left(s^{L} / k\right)-\bar{u}^{A}\right]-\varepsilon h x^{-1}\left(\bar{u}^{B}-\bar{u}^{A}\right)+\varepsilon x\left(\mathbb{E} W^{B}-\mathbb{E} W^{A}\right)-\varepsilon h \mathbb{E} W^{A} \\
\Rightarrow \\
u(q)=\bar{u}^{A}+h\left(\mathbb{E} W^{A}-k\left[\hat{u}\left(s^{L} / k\right)-\bar{u}^{A}\right]\right)-\left(1-h x^{-1}\right)\left(\bar{u}^{B}-\bar{u}^{A}\right)
\end{gathered}
$$

Using (15), rewritten as

$$
\mathbb{E} W^{B}-k\left[\hat{u}\left(s^{L} / k\right)-\bar{u}^{B}\right]=\mathbb{E} W^{A}-k\left[\hat{u}\left(s^{L} / k\right)-\bar{u}^{A}\right]+x^{-1}\left(\bar{u}^{B}-\bar{u}^{A}\right),
$$

we can rewrite

$$
\begin{gathered}
u(q)=\bar{u}^{A}+h\left(\mathbb{E} W^{B}-k\left[\hat{u}\left(s^{L} / k\right)-\bar{u}^{B}\right]\right)+h\left(\mathbb{E} W^{A}-\mathbb{E} W^{B}+\bar{u}^{B}-\bar{u}^{A}\right)-\left(1-h x^{-1}\right)\left(\bar{u}^{B}-\bar{u}^{A}\right) \\
\Rightarrow \\
u(q)=\bar{u}^{A}+h\left(\mathbb{E} W^{B}-k\left[\hat{u}\left(s^{L} / k\right)-\bar{u}^{B}\right]\right)
\end{gathered}
$$

where the last two bracketed terms cancel out in the next to the last line. The last term seems to depend on outside option $\bar{u}^{B}$, but by use of Remark 1, we see it measures scarcity which only depends on beliefs $\left(\alpha, s^{L}\right)$. Using $h=\alpha q$ :

$$
\begin{aligned}
\alpha\left(\mathbb{E} W^{B}-k\left[\hat{u}\left(s^{L} / k\right)-\bar{u}^{B}\right]\right) & =-\int_{s^{L}}^{\infty} k\left[\hat{u}(s / k)-\bar{u}^{B}\right] f_{s}\left(s ; s^{L}, \alpha\right) d s+\left[k\left(\hat{u}(s / k)-\bar{u}^{B}\right) f\left(s ; s^{L}, \alpha\right)\right]_{0}^{\infty} \\
& =\int_{s^{L}}^{\infty} \hat{u}^{\prime}(s / k) f\left(s ; s^{L}, \alpha\right) d s \\
& =\mathbb{E}\left[\hat{u}^{\prime}(s / k) \mid s^{L}\right] \\
& =\lambda\left(\alpha, s^{L}\right)
\end{aligned}
$$

leading to the indifference condition,

$$
u(q)=\bar{u}^{A}+\lambda q
$$

showing that the buyer's stopping condition has not changed. 
The seller's separation decision can be formulated as an opt-out problem where the seller chooses whether to supply $q^{A}$, or more, at any given time, and for how long. Supposing the seller with initial $s_{0}$ keeps the buyer indifferent as long as the state is $A$, so it will supply $q^{A}$. Then, for a given future time $T$ for opting out, the expected payoff is:

$$
V\left(s_{0}\right)=\int_{0}^{T} x e^{-x t}\left[\pi\left(q^{A}\right) t-V\left(s_{0}-q^{A} t\right)\right] d t+e^{-x T}\left[\pi\left(q^{A}\right) T-V\left(s_{0}-q^{A} T\right)\right]
$$

The first part on the right captures the outcomes where the better outside option and thus stopping by the buyer arrives at time $t$ before the planned stopping time by the seller $T$, with probability $x e^{-x t}$ and payoff $\pi\left(q^{A}\right) t-V\left(s_{0}-q^{A} t\right)$. The second part corresponds to events where the seller stops first at time $T$ with probability $e^{-x T}$. Yet, the first order condition for the opt-out time is precisely as in (17), so that the seller's separation curve $\mathcal{S}\left(s^{L}\right)$ remains the same. The new substitute arrival is exogenous to the seller's stopping decision and thus the seller's separation type is unchanged. Q.E.D.

\section{Proof of Proposition 7}

By Proposition 6, we can analyze the event as if it was unanticipated; the equilibrium in state A progresses as in the absence of state B. We have characterized the continuation equilibrium: from Proposition 3, the informed party triggers stopping by a supply disruption conditional on reaching the privately known stopping level for the stock. If such this level is not reached, state B arrives and the buyer stops. By the properties of the distribution $F(s ; \cdot)$, there is positive probability for the event that the seller has more than $k q^{m}$, in which case some stock will be left unused. Q.E.D.

\section{References}

[1] Akerlof, G. 1970: The Market for Lemons: Quality Uncertainty and the Market Mechanism, Quarterly Journal of Economics, 84, 488- 500.

[2] Ausubel M. L., P. Cramton, and R.J. Deneckere (2002), Bargaining with Incomplete Information, In Handbook of Game Theory vol. 3, R.J. Aumann and S. Hart (Eds.), Amsterdam, Elsevier Science B.V., Chapter 50.

[3] Brown, J.R., (1963), Nitrate Crises, Combinations, and the Chilean Government in the Nitrate Age, The Hispanic American Historical Review, Vol. 43, No. 2, 230-246 
[4] Crawford, V.P. and J. Sobel (1982), Strategic Information Transmission, Econometrica, vol. 50(6), pages 1431-51, November.

[5] Coase, R. H. (1972), "Durability and Monopoly", Journal of Law and Economics, $15,143-49$.

[6] Cordella, D, J.-O. Drangerta, and S. Whiteb (2009). The story of phosphorus: Global food security and food for thought, Global Environmental Change Volume 19, Issue 2, Pages 292- 305

[7] Dasgupta, P. S., and G. M. Heal. 1974. "The Optimal Depletion of Exhaustible Resources", Review of Economic Studies 41, 3-38.

[8] Dasgupta, P. S., and G. M. Heal. 1979. "Economic Theory and Exhaustible Resources ", Cambridge: Cambridge Univ. Press.

[9] Daley, B., and B. Green, Waiting for News in the Market for Lemons, Econometrica, Vol. 80, No. 4 (July, 2012), 1433- 1504.

[10] Gallini, N., T. Lewis, and R.Ware (1983), Strategic Timing and Pricing of a Substitute in a Cartelized Resource Market, The Canadian Journal of Economics, Vol. 16, No. 3., 429-446.

[11] Gerlagh, R., and M. Liski, Strategic Resource Dependence, FEEM Working Paper No. 72.2008 .

[12] Hotelling, H. (1931), The economics of exhaustible resources, Journal of Political Economy 39, 137-175.

[13] Dasgupta, P., R. Gilbert, and J. Stiglitz (1983), Strategic Considerations in Invention and Innovation: The Case of Natural Resources, Econometrica 51, 1439-1448.

[14] Deneckere R.J., and M.-Y. Liang (2006), Bargaining with Interdependent Values, Econometrica 74, 1309-1364.

[15] Dutta, P. (1991), What Do Discounted Optima Converge To? A Theory of Discount Rate Asymptotics in Economic Models, Journal of Economic Theory 55, 64-94.

[16] Fudenberg, D., and J. Tirole (1983), Sequential Bargaining with Incomplete Information, Review of Economic Studies 50, 221-247. 
[17] Gerlagh, R., and M. Liski (2011). Strategic resource dependence, Journal of Economic Theory, Volume 146, Issue 2, Pages 699-727.

[18] Gul, F., H. Sonnenschein, and R. Wilson (1986), "Foundations of Dynamic Monopoly and the Coase Conjecture", Journal of Economic Theory 39, 155-190.

[19] Harris, C., and J. Vickers (1995), Innovation and Natural Resources: A Dynamic Game with Uncertainty, The RAND Journal of Economics 26, 418-430.

[20] Harstad B., and Liski M. (2013) Games and Resources. In: Shogren, J.F., (ed.) ÒEncyclopedia of Energy, Natural Resource, and Environmental EconomicsÓ, Vol. 2, pp. 299-308 Amsterdam: Elsevier.

[21] Hoel, M. (1983) Monopoly resource extractions under the presence of predetermined substitute production, Journal of Economic Theory Volume 30, Issue 1, June 1983, Pages 201- 212.

[22] Hörner, J., and Kamien, M. (2004), Coase and Hotelling: A Meeting of the Minds, Journal of Political Economy, vol. 112, no. 3.

[23] Karp, L. and D. Newbery (1993), Intertemporal Consistency Issues in Depletable Resources, In Handbook of Natural Resource and Energy Economics vol. 3, A.V. Kneese and J.L. Sweeney (Eds.), Amsterdam, North Holland.

[24] M. Kemp, How to eat a cake of unknown size, in: M. Kemp (Ed.), Three Topics in the Theory of International Trade, North-Holland, Amsterdam, 1976.

[25] Keyzer, M. (2010). Towards a Closed Phosphorus Cycle,Volume 158, Issue 4, pp 411-425.

[26] Kremer, I., and A. Skrzypacz (2007), Dynamic signaling and market breakdown, Journal of Economic Theory 133, 58 - 82.

[27] Kumar, R. (2005), How to eat a cake of unknown size: A reconsideration, Journal of Environmental Economics and Management 50, 408-421

[28] Leigh, G. J., (2004) The world's greatest fix: a history of nitrogen and agriculture, Oxford University Press 
[29] Lewis, T., R. Lindsey, and R. Ware (1986), Long-Term Bilateral Monopoly: The Case of an Exhaustible Resource, The RAND Journal of Economics, Vol. 17, No. 1., 89-104.

[30] Nöldeke, G., and van Damme, E. (1990) Signalling in a Dynamic Labour Market, Review of Economic Studies, Wiley Blackwell, vol. 57(1), pages 1-23, January.

[31] Janssen, M.C. W., and S. Roy (2002). Dynamic Trading in a Durable Good Market with Asymmetric Information, International Economic Review, vol. 43(1), pages 257-282, February.

[32] Maskin, E., and D. Newbery (1990), Disadvantageous Oil Tariffs and Dynamic Consistency, The American Economic Review 80, No. 1 , 143-156.

[33] Mokyr, J. (1998), "The Second Industrial Revolution, 1870-1914." in Valerio Castronovo, ed., Storia dell'economia Mondiale. Rome: Laterza publishing. Donwloaded from http://faculty.wcas.northwestern.edu/ jmokyr/castronovo.pdf

[34] Montéon, M. (1975), The British in the Atacama Desert: The Cultural Bases of Economic Imperialism, The Journal of Economic History, Vol. 35, No. 1, 117-133

[35] Newbery, D (1981), Oil Prices, Cartels, and the Problem of Dynamic Inconsistency The Economic Journal. Vol. 91, No. 363 (Sep., 1981), pp. 617-646

[36] Nordhaus; W.D. (1973), The Allocation of Energy Resources, Brookings Papers on Economic Activity 1973 (3): 529-570.

[37] Pindyck, Robert S, 1980. "Uncertainty and Exhaustible Resource Markets," Journal of Political Economy, University of Chicago Press, vol. 88(6), pages 1203-25, December.

[38] Simmons, M.R. (2005) Twilight in the Desert: The Coming Saudi Oil Shock and the World Economy, John Wiley \& Sons., New Jersey.

[39] Smil, V. (2001) Enriching the Earth: Fritz Haber, Carl Bosch and the Transformation of World Agriculture, Cambridge, MA.

[40] Spence, M. A. 1973: Job Market Signaling, Quarterly Journal of Economics, 90, 225- 243. 
[41] Swinkels, J.M, (1999) Education Signalling with Preemptive Offers, Review of Economic Studies, Wiley Blackwell, vol. 66(4), pages 949-70, October.

[42] Schweinzer, P, (2010). Sequential bargaining with common values, Journal of Mathematical Economics 46 (2010) 109- 121.

[43] Whitbeck, R. H. (1931), Chilean Nitrate and the Nitrogen Revolution, Economic Geography, Vol. 7, No. 3, 273-283 